\title{
A CFD study of the parameters influencing heat transfer in microchannel slug flow boiling
}

\author{
M. Magnini*, J. R. Thome \\ Laboratory of Heat and Mass Transfer (LTCM), Ecole Polytechnique Fédérale de \\ Lausanne (EPFL), EPFL-STI-IGM-LTCM, Station 9, CH-1015 Lausanne, Switzerland
}

\begin{abstract}
Boiling heat transfer in microchannels has been investigated extensively in the last two decades. However, most results from independent experimental studies display a substantial disagreement on the influence of the flow conditions and mechanisms on the heat transfer performance. The objective of the present paper is to clarify by means of CFD simulations the effect of the primary flow parameters on the saturated flow boiling heat transfer performance of a slug flow within a horizontal, circular microchannel. The vapor quality, heat flux, mass flux, channel diameter, bubble frequency, and saturation temperature are the parameters under analysis. The numerical model is based on the volume of fluid method included within ANSYS Fluent v. 14.5, which is here augmented by implementing ad-hoc models to calculate the surface tension force, the mass and energy transfer at the interface due to phase change, and to generate a continuous stream of bubbles with an arbitrary frequency. The high fidelity of the numerical framework provides unprecedented insight on the effect of these flow parameters on the bubble
\end{abstract}

\footnotetext{
${ }^{*}$ Corresponding author. Tel.: +41 0216937343.

Email address: mirco.magnini@epfl.ch (M. Magnini)
} 
dynamics and heat transfer performance, which are tightly interrelated in microchannel slug flow boiling. In principle, the heat transfer performance is enhanced by flow conditions promoting a thin liquid film surrounding the elongated vapor bubbles and by the flow within short liquid slugs between the bubbles. Furthermore, numerous interesting secondary effects driven by the different flow conditions are observed and described according to the underlying flow dynamics: time-dependent interfacial waves on the bubble surface which significantly reduce the local film thickness; the response time of the channel wall temperature to changes of the flow boundary conditions at different channel sizes; the liquid film thickness dependence on the bubble length and on the liquid slug length; the heat transfer dependence on heat flux via the bubble generation frequency. A dimensional analysis is finally conducted in order to identify the nondimensional groups relevant to the microchannel bubble dynamics and heat transfer in the slug flow regime.

Keywords: slug flow, heat transfer, evaporation, microchannel, bubble dynamics

\section{Introduction}

Flow boiling and heat transfer in microchannels are receiving increasing attention as the most advantageous cooling process for miniaturized electronic devices. The use of latent heat and the relatively large liquid-vapor interface area promoted by microscale geometries enhance the mass transfer associated with the evaporation process, and hence the thermal performance of the cooling system when compared to traditional single-phase based technologies [1]. 
Due to the large variety of applications of two-phase flows in narrow channels, originally in nuclear reactor cooling systems and, at a later stage, in the thermal management of Micro Electronic and Mechanical Systems (MEMS) $[2,3,4]$ as well as microbiochemical reactors $[5,6,7]$, in the last two decades leading laboratories in the world have published a conspicuous number of experimental studies covering two-phase flow patterns, pressure drop, heat transfer, void fraction, and critical heat flux in microscale conditions. However, this common effort has not yet led to broadly accepted unified descriptions of the relevant microscale two-phase flow phenomena, of the governing fluid dynamics and heat transfer mechanisms, and hence of the influence of the flow conditions on the cooling capability of the flow. As a matter of fact, experimental measurements from independent studies display very diverse trends of the heat transfer coefficient, which is the parameter utilized to quantify the thermal efficiency of the flow, versus the vapor quality for different values of heat flux, mass flux, and saturation pressure. Agostini and Thome [8], Tibiriçá and Ribatski [9], Baldassari and Marengo [10], Wang and Wang [11] gathered a large number of experimental data from the open literature in the attempt to summarize the most frequently observed trends. Conflicting trends under similar operating conditions were reported and these were mainly attributed to: (i) different flow patterns, which were unknown before flow visualization began to be joined to heat transfer measurements, as flow patterns significantly influence the boiling heat transfer coefficient trends in microchannels [12]; (ii) flow instability and pressure fluctuations related to the upstream bubble formation process in both single [13] and multiple [14] parallel microchannels, which can induce 
wall temperature oscillations of several Kelvin; (iii) the data reduction procedure and, in particular, the way the profile of the local saturation pressure along the microchannel streamwise direction is reconstructed, as it directly influences the computed values of the heat transfer coefficient [15].

Within microchannels, the increased importance of capillary forces promotes a higher segregation between the liquid and vapor phases compared to macroscale flows, and therefore the two-phase flow structures characterizing the observed flow regimes, usually identified as bubbly, slug, annular and post-dryout mist flow, appear rather simplified. As a consequence, mechanistic models attempting to estimate the flow boiling heat transfer coefficient by accounting for the actual geometry of the interface have proven to perform better than empirical correlations when utilized to predict experimental data from independent studies $[16,17,18,19]$, although still showing a large degree of uncertainty.

However, it is worth noting that the lack of agreement on the heat transfer performance trends mentioned above, together with the technical limitations of the current experimental measurement techniques with respect to temporal and spatial resolutions, make the deduction of the underlying physics from the available two-phase flow data a complex and somewhat subjective task, which in turn limits the development of more reliable mechanistic models to predict the relevant thermal-hydraulic parameters.

Unlike experimental studies, CFD simulations are more able to provide detailed information on the local hydrodynamics and heat transfer of boiling two-phase flows in the microscale, and hence can be utilized as an additional research tool. For example, Mukherjee et al. [20] analyzed the growth of 
a vapor bubble under flow boiling conditions in a microchannel of square cross-section and the associated wall heat transfer. Fang et al. [21] carried out simulations of the flow boiling of multiple bubbles in a vapor-venting microchannel to examine flow patterns and pressure drop. Zhuan and Wang [22] explored the effect of bubble lift-off size, heat flux, mass velocity, frequency of bubble generation and fluid properties on flow patterns and related transitions within circular microchannels. Pattamatta et al. [23] studied the conditions promoting Taylor bubbles coalescence in a square microchannel. Magnini et al. [24, 25] investigated the role of the liquid film surrounding elongated bubbles and of the recirculation patterns in the liquid slug trapped between successive bubbles on the thermal performance of elongated bubble flows in circular microchannels. Pan et al. [26] performed 3D simulations to analyze the dynamics of a single bubble and heat transfer during flow boiling in a square microchannel.

The objective of the present paper is to investigate and clarify by means of two-phase flow CFD simulations the influence of selected flow parameters, namely vapor quality, wall heat flux, channel diameter, mass flux, saturation temperature and bubble frequency, on the saturated flow boiling heat transfer performance of a slug flow within a horizontal, circular microchannel. This study utilizes the refrigerant R245fa as the working fluid, and it covers channel diameters from $0.3 \mathrm{~mm}$ to $0.7 \mathrm{~mm}$, mass fluxes from $400 \mathrm{~kg} /\left(\mathrm{m}^{2} \mathrm{~s}\right)$ to $700 \mathrm{~kg} /\left(\mathrm{m}^{2} \mathrm{~s}\right)$, heat fluxes from $5 \mathrm{~kW} / \mathrm{m}^{2}$ to $20 \mathrm{~kW} / \mathrm{m}^{2}$, saturation temperatures from $10^{\circ} \mathrm{C}$ to $50^{\circ} \mathrm{C}$, and bubble frequencies from $70 \mathrm{~Hz}$ to $270 \mathrm{~Hz}$. The trends of the heat transfer coefficient obtained with the numerical model 
are explained in conjunction with the observed dynamics of the growing bubbles, by the liquid film trapped between the bubble and the channel wall, and by the flow field in the liquid slug trapped between two successive bubbles, and thus allow to deduce the most influential fluid dynamic features on the heat transfer mechanisms. Similarities and deviations between the present numerical results and experimentally observed trends for the flow regime of interest here are highlighted and discussed. Finally, a dimensional analysis is proposed to identify the nondimensional groups relevant to the microchannel bubble dynamics and heat transfer.

The commercial CFD software ANSYS Fluent v. 14.5 is presently adopted to perform the numerical simulations, and its built-in Volume Of Fluid (VOF) method [27] is utilized to capture the interface dynamics. The standard CFD package has been presently augmented by an improved surface tension force estimation model based on the Height Function (HF) technique, by an evaporation model based on the work of Hardt and Wondra [28], and by a routine to generate vapor bubbles with a prescribed initial volume and frequency. These have been implemented as self-developed User-Defined Functions (UDF).

This paper is organized as follows: in Section 2, a literature review of the experimentally observed heat transfer coefficient trends is undertaken; in Section 3, the numerical model is described; in Section 4, the results of the parametric study are presented and discussed; in Section 5, a dimensional analysis of the parameters governing heat transfer in microchannel slug flow is carried out. 


\section{Heat transfer coefficient trends observed in experiments for slug flow boiling in microchannels}

The effects of vapor quality, heat flux, mass flux, channel diameter, and saturation pressure on the heat transfer coefficient trends observed in experiments for slug flow boiling in microchannels are discussed below. In the two-phase flow literature, the heat transfer coefficient is usually represented as a function of the vapor quality $x$, which can range from negative values (subcooled boiling) to unity. In the absence of flow visualization or flow pattern maps to discriminate the different two-phase flow regimes in the original published data, the range of vapor qualities corresponding to the slug flow regime is unknown. As a rule of thumb, results of heat transfer coefficient measurements from the literature for the interval $x=[0,0.2]$ are considered in the remainder of this section, unless more precise information about flow patterns are provided in the original publication.

In the low vapor quality region of interest here, the heat transfer coefficient is independent of the vapor quality in many different studies $[29,30$, $31,32,33,34]$, although monotonic ascending [35] as well as monotonic descending [36] trends were also detected. In a few studies, the heat flux acted as an influential parameter. The heat transfer coefficient was observed to increase monotonically with $x$ (always within the $[0,0.2]$ range) at lower heat fluxes (usually below about $50 \mathrm{~kW} / \mathrm{m}^{2}$ ), but to decrease with $x$ at higher heat fluxes [37, 38, 39, 40,41]. The latter was often ascribed to intermittent dryout of the liquid film surrounding the elongated bubbles and promoted by

higher thermal loads. A more complex behavior was found, see the sketch in Fig. 1: the heat transfer coefficient rose and then dropped showing a local 


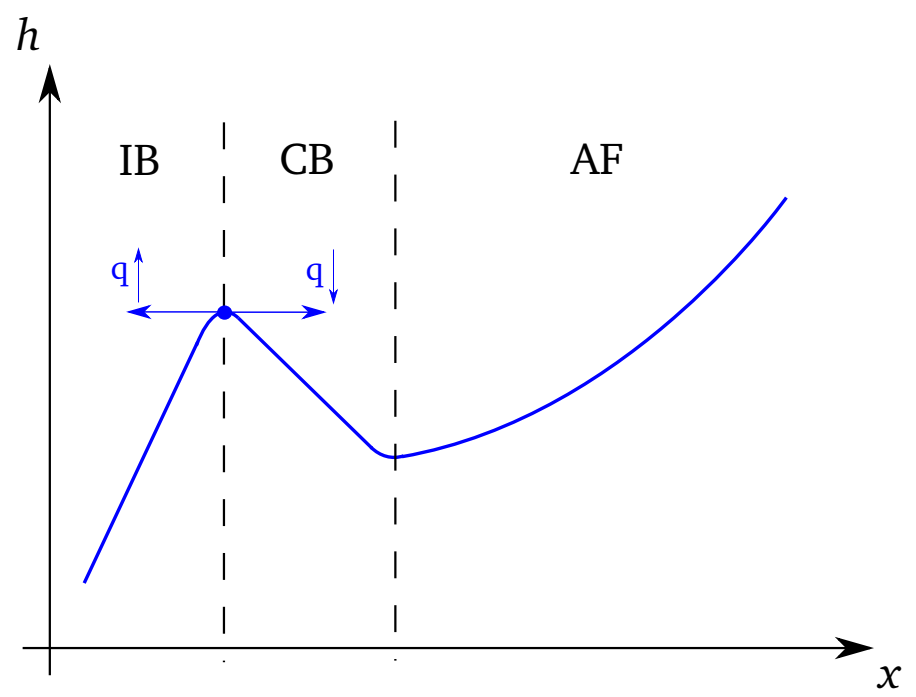

Figure 1: Sketch of the heat transfer coefficient trend observed in experiments at different flow regimes: IB - isolated bubble, $\mathrm{CB}$ - coalescing bubble, AF - annular flow. The heat flux $q$ acts as a parameter for the IB to CB transition.

maximum at very low vapor qualities ( $x$ below 0.1 ), afterwards it rose again as $x$ was further increased as observed in $[12,42,14]$. This trend has been related to peculiar flow pattern transitions: Revellin et al. [43] measured the bubble frequency against the vapor quality after the heated section of a microchannel and noticed that it presented a maximum in the very low vapor quality region ( $x<0.05$ under their working conditions), which they identified as the transition between an isolated bubble (IB) and a coalescing bubble $(\mathrm{CB})$ regime, split at the peak in bubble frequency that they observed. Later on, Ong and Thome [44, 42] correlated flow visualization and heat transfer measurements and observed that the local maximum of the heat transfer coefficient at vapor qualities below 0.1 again matched the transition between the two flow patterns mentioned above. The increasing 
trend of the heat transfer coefficient with $x$ in the IB regime was related to the increasing bubble frequency, while the successive fall in the $\mathrm{CB}$ regime was ascribed to intermittent dryout at the tail of the very elongated bubbles. The subsequent local minimum and then growth of the heat transfer coefficient was attributed to another flow pattern transition, from slug to annular flow, itself ruled by different heat transfer mechanisms. Furthermore, at high heat fluxes the IB regime was suppressed (the IB to CB transition moved to lower values of $x$, as depicted in Fig. 1) and the slug to annular flow transition occurred at very low vapor qualities, so that only descending and then ascending trends with $x$ were observed.

Most of the experimental studies from the open literature show that, in the range of $x$ considered here, the heat transfer coefficient increases with the heat flux, while a more complex behavior was reported by Agostini et al. [38] at high mass fluxes. While numerous microchannel flow boiling correlations attributed this heat flux influence to nucleate boiling, these are unsubstantiated claims disproved in experimental studies including flow visualization, i.e. typically there is only one (or several) nucleating site providing all the downstream bubbles. In the cyclical passage of elongated bubbles and liquid slugs which is characteristic of the slug flow pattern, the maximum heat transfer rate is consider to be achieved during the transit of the elongated bubble and the minimum in correspondence of the liquid slug [17]. This has been more recently confirmed by the high resolution heat transfer measurements performed by Rao and Peles [45] for flow boiling in microchannels. Higher heat fluxes lead to: (i) higher bubble nucleation frequency [46] and hence shorter liquid slugs, which is a configuration known to enhance the 
bubble-liquid slug unit time-averaged heat transfer coefficient [47]; and (ii) to higher evaporation rates which promote thinner liquid films to exist [17], and hence higher heat transfer rates in the region occupied by the elongated bubble.

The heat transfer coefficient often exhibited little mass flux dependence $[30,32,48,33,49,50,13]$, which led many authors to the conclusion that nucleate boiling, rather than convective boiling, was the dominant heat transfer mechanism within microchannels. However, contrasting trends were also measured, with the heat transfer coefficient either decreasing [35, 37, 41] or increasing $[39,34]$ by an increment of mass flux. The former could be attributed to an earlier coalescence of bubbles and therefore occurrence of intermittent dryout. The latter can be explained by an augmentation of the bubble nucleation frequency induced by the higher mass flow rate which promotes bubble detachment due to drag force effects, which is an interpretation compatible with an increased bubble count measured by Revellin et al. [43] when increasing the mass flux. Liu et al. [51] reported that the parametric dependence of the heat transfer coefficient on mass flux and heat flux varies substantially according to the importance of upstream inlet compressibility, which may yield different flow boiling heat transfer trends. Interestingly, Ong and Thome [42] observed that the slug to annular flow transition line was shifted toward lower vapor qualities as mass flux grew, thus suppressing the IB and $\mathrm{CB}$ regimes and expanding the vapor quality range where annular flow occurred. This further highlights the importance of an accurate identification of the underlying flow patterns when interpreting heat transfer coefficient trends with $x$. 
In general, the experimental studies show that a reduction of the channel size enhances the heat transfer performance of the slug flow regime, as a consequence of the thinner liquid film and thus higher evaporation rates [52]. However, an opposite trend was found in the low vapor quality range by Ong and Thome [42] (channel diameters from $3 \mathrm{~mm}$ to $1 \mathrm{~mm}$ ), while a negligible influence of the channel diameter was detected by Huo et al. [48] (channel diameters from $4.26 \mathrm{~mm}$ to $2 \mathrm{~mm}$ ).

The saturation pressure is generally observed to enhance the heat transfer performance, because (i) the bubble nucleation frequency is augmented and (ii) the vaporization of the liquid film is promoted by the lower latent heat of vaporization, although different behaviors were also reported [53, 38]. Interestingly, simulations run with the Thome et al. [17] three-zone boiling heat transfer model showed that the heat transfer coefficient dropped with an increase in the saturation pressure in the vapor quality range corresponding to the IB regime. This effect is reasonable when considering that a larger saturation pressure reduces the liquid-to-vapor density ratio, thus yielding shorter bubbles and therefore decreasing the liquid film residence time; however, this model by itself is not able to correctly predict all the trends noted above.

The review of the experimental results for the boiling heat transfer in microchannel slug flows developed above emphasizes two important aspects: (i) the lack of agreement about the effect of some flow parameters on the heat transfer coefficient, in particular with respect to vapor quality, mass flux and saturation pressure; (ii) the microchannel two-phase flow configuration under 
evaporating conditions is a complex result of bubble nucleation, growth, and detachment phenomena, combined with fluid dynamics and heat transfer mechanisms which are peculiar of confined bubble flows, such that a better understanding of the effect of each flow parameter would be possible only by analyzing these two stages separately. To the end of shedding light on these aspects, the present work investigates the effect of the main flow parameters on slug flow bubble dynamics and heat transfer regardless of the bubble generation process. This is done in the present CFD simulations by setting and changing the bubble initial volume and frequency independently of the other flow conditions imposed.

\section{Numerical model}

\subsection{Governing equations}

When the VOF method is utilized to capture the interface dynamics, a volume fraction $\alpha$ is defined to identify each phase on a Eulerian computational grid. It represents the ratio of the cell volume occupied by the primary phase and hence ranges from 0 to 1 . By means of the volume fraction field, the phases are treated as a single fluid whose properties change abruptly across the vapor-liquid interface, according to the cell value of the volume fraction $\alpha$.

The single-fluid flow equations include the volume fraction, continuity, momentum, and energy equations, which for a Newtonian fluid and incompressible flow under phase change can be written as:

$$
\frac{\partial \alpha}{\partial t}+\nabla \cdot(\alpha \boldsymbol{u})=\frac{1}{\rho_{v}} \dot{m}|\nabla \alpha|
$$




$$
\begin{gathered}
\nabla \cdot \boldsymbol{u}=\left(\frac{1}{\rho_{v}}-\frac{1}{\rho_{l}}\right) \dot{m}|\nabla \alpha| \\
\frac{\partial(\rho \boldsymbol{u})}{\partial t}+\nabla \cdot(\rho \boldsymbol{u} \cdot \boldsymbol{u})=-\nabla p+\nabla \cdot\left[\mu\left(\nabla \boldsymbol{u}+\nabla \boldsymbol{u}^{T}\right)\right]+\rho \boldsymbol{g}+\boldsymbol{F}_{\boldsymbol{\sigma}} \\
\frac{\partial\left(\rho c_{p} T\right)}{\partial t}+\nabla \cdot\left(\rho c_{p} \boldsymbol{u} T\right)=\nabla \cdot(\lambda \nabla T)-\dot{m}\left[h_{l v}-\left(c_{p, v}-c_{p, l}\right) T\right]|\nabla \alpha|
\end{gathered}
$$

where $\boldsymbol{u}$ is the fluid velocity, $\rho$ the density, $\dot{m}$ the mass flux due to phase change, $p$ the pressure, $\mu$ the dynamic viscosity, $\boldsymbol{g}$ the gravity acceleration, $\boldsymbol{F}_{\boldsymbol{\sigma}}$ the surface tension force vector, $T$ the temperature, $c_{p}$ the constant pressure specific heat, $\lambda$ the thermal conductivity, and $h_{l v}$ is the latent heat of vaporization. The subscripts $v$ and $l$ identify vapor and liquid properties, respectively.

The surface tension force is formulated according to the Continuum Surface Force (CSF) method proposed by Brackbill et al. [54]:

$$
\boldsymbol{F}_{\boldsymbol{\sigma}}=\frac{2 \rho}{\rho_{l}+\rho_{v}} \sigma \kappa \nabla \alpha
$$

where $\sigma$ is the surface tension coefficient, here considered constant, and $\kappa$ is the local interface curvature, which is here estimated by means of a Height Function (HF) algorithm. This algorithm is not available in the default solver and has been presently implemented as a User-Defined Function. The interphase evaporation mass flux is calculated by adopting the model of Tanasawa [55], which accounts for the deviation of the interface temperature from the saturation temperature and contains a linear dependence of the mass flux on the interface superheating:

$$
\dot{m}=\frac{2 \gamma}{2-\gamma}\left(\frac{M}{2 \pi R_{g}}\right)^{1 / 2} \frac{\rho_{v} h_{l v}\left(T_{l v}-T_{s a t}\right)}{T_{s a t}^{3 / 2}}
$$


where $\gamma$ is the evaporation coefficient, here set to 1 according to preliminary test benchmarks [24], $M$ is the molecular weight of the fluid, $R_{g}$ the universal gas constant, while $T_{l v}$ and $T_{\text {sat }}$ denote the interface temperature and the saturation temperature, respectively. In this work, the evaporation source terms are made smoother across the interface according to the procedure proposed by Hardt and Wondra [28]. This entire evaporation model has been implemented as a UDF within the main code. Details of the surface tension and evaporation model algorithms utilized here, and related validation benchmarks, are provided in Magnini [56] and Magnini et al. [24].

\subsection{Flow solver}

The commercial CFD solver ANSYS Fluent release 14.5 is utilized to solve the flow problem. It adopts a finite-volume discretization of the flow equations in a co-located grid arrangement. The volume fraction equation is integrated in time with a first order explicit scheme and the PLIC technique (Piecewise Linear Interface Calculation) [57] is employed to discretize the convective term of Eq. (1). An implicit first order time scheme is used to solve the momentum and energy conservation equations and third order schemes are used for their spatial discretization. The PISO algorithm (Pres-

sure Implicit Splitting of Operators) [58] is chosen for the pressure-velocity coupling. The built-in PRESTO option (Pressure Staggering Option), which solves the pressure correction equation for a staggered control volume thus providing face-centered values of the pressure without the need for interpolations, is adopted. A variable time-step is computed by the solver according to a maximum Courant number of 0.5. The default Gauss-Seidel iterative solver with an Algebraic Multigrid technique is used to solve the system of 
equations, and the convergence criterion is set to a value of $10^{-6}$ of the normalized absolute residuals for all the flow equations. More details about the present selections of the discretization methods are provided in Magnini et al. $[25]$.

\subsection{Set-up of simulations}

A slug flow within a horizontal circular microchannel under saturated flow boiling conditions is simulated here for 13 different sets of flow conditions. The microchannel is modelled as a two-dimensional axisymmetric domain of diameter $D$. It is split into an initial adiabatic section of $8 D$ in length, followed by a heated section of $22 D$ length, which is the object of the study, and it ends with a terminal adiabatic section of variable length, from $15 D$ to $30 D$, according to the expected terminal length of the bubbles. A continuous stream of bubbles is generated by patching elongated vapor bubbles of initial length $3 D$ (and volume $0.526 \pi D^{3}$ ) at the upstream initial adiabatic section of the channel with a prescribed frequency $f$. This allows us to decouple the bubble generation phenomenon from the successive flow boiling process, such that the frequency effects on heat transfer can be studied independently of the other flow parameters. The operating fluid is refrigerant R245fa. Figure 2 provides a schematic of the initial and boundary conditions set in the simulations. At the channel inlet, liquid at the saturation temperature flows with average velocity $U_{l}$, which is calculated according to the desired value of the mass flux as $U_{l}=G / \rho_{l}$. A constant and uniform heat flux is applied at the wall of the heated region. A no-slip boundary condition is set at the channel wall. At the channel outlet, a zero gradient boundary condition is imposed for both velocity and temperature, along with a constant value for the pressure. 


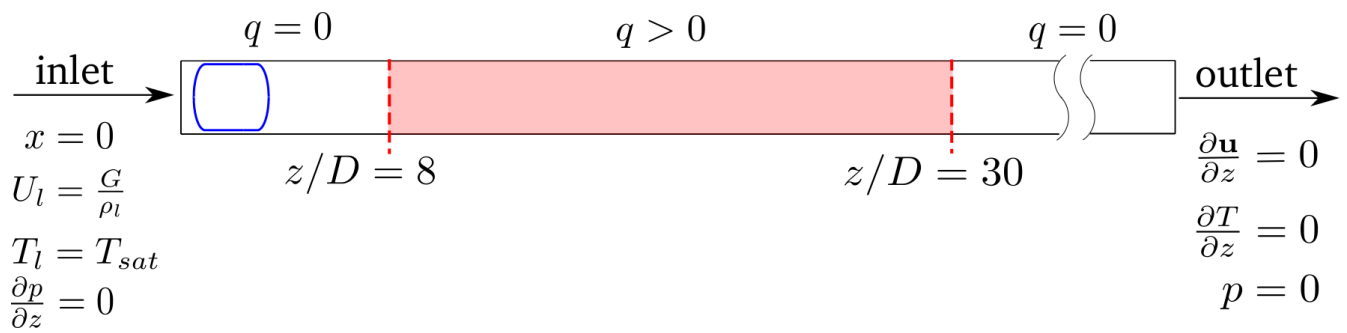

Figure 2: Schematic of initial and boundary conditions for the simulations. The bubble profile is represented by the blue line profile at the upstream of the channel. Figure is not to scale.

The velocity and temperature fields at the beginning of the simulation are obtained from a preliminary liquid-only steady-state simulation run under the same flow conditions.

Table 1 summarizes the flow parameter sets in the 13 simulations which are presented in this work. The flow conditions set for Case 1 represent the reference situation, then the effects of heat flux (runs 1-3), mass flux (1, 4, $5)$, bubble frequency $(1,6-9)$, channel diameter $(1,10,11)$, and saturation temperature $(1,12,13)$, are investigated separately by varying the flow parameters one at a time from the reference Case 1 . Note that the value of the bubble frequency for each simulation run is chosen in such a way that the initial length $L_{l}$ of the liquid slug trapped between two consecutive bubbles is about $6 D$, except for cases 6 to 9 where the frequency is varied to achieve different liquid slug lengths (from $0.5 D$ to $10 D$, see Table 1 ). The inlet vapor quality for all the test runs is on the order of $10^{-3}$, while the maximum exit vapor quality is 0.02 . The empirical correlation derived by Ong and Thome [44] to predict flow regime transitions under operating conditions similar to 
those of interest here, yields an isolated to coalescing bubble flow transition at $x=0.019$. Hence, the range of vapor quality tested in this work well represents those of a slug flow in the isolated bubble regime.

The computational mesh for the microchannel is composed of square cells of size $D / 300$. According to the grid convergence analysis reported in [24], this grid resolution is sufficient to achieve mesh-independent results. Every simulation case involved about 2 million mesh cells and required about 150000 time steps to run more than $100 \mathrm{~ms}$ of simulation time. Computations were performed on the EPFL Bellatrix cluster, having computing nodes of 2 Sandy

\begin{tabular}{|c||c|c|c|c|c|c|}
\hline Case & $D[\mathrm{~mm}]$ & $T_{\text {sat }}\left[{ }^{\circ} \mathrm{C}\right]$ & $G\left[\mathrm{~kg} /\left(\mathrm{m}^{2} \mathrm{~s}\right)\right]$ & $q\left[\mathrm{~kW} / \mathrm{m}^{2}\right]$ & $f[\mathrm{~Hz}]$ & $L_{l} / D[-]$ \\
\hline 1 & 0.5 & 31 & 550 & 5 & 104 & 6 \\
2 & 0.5 & 31 & 550 & 10 & 104 & 6 \\
3 & 0.5 & 31 & 550 & 20 & 104 & 6 \\
4 & 0.5 & 31 & 400 & 5 & 76 & 6 \\
5 & 0.5 & 31 & 700 & 5 & 143 & 6 \\
6 & 0.5 & 31 & 550 & 5 & 269 & 0.5 \\
7 & 0.5 & 31 & 550 & 5 & 209 & 1.5 \\
8 & 0.5 & 31 & 550 & 5 & 157 & 3 \\
9 & 0.5 & 31 & 550 & 5 & 72 & 10 \\
10 & 0.3 & 31 & 550 & 5 & 177 & 6 \\
11 & 0.7 & 31 & 550 & 5 & 78 & 6 \\
12 & 0.5 & 10 & 550 & 5 & 104 & 6 \\
13 & 0.5 & 50 & 550 & 5 & 114 & 6 \\
\hline
\end{tabular}

Table 1: Operating conditions for the present simulation runs. 
Bridge processors running at $2.2 \mathrm{GHz}$, with 8 cores each and $32 \mathrm{~GB}$ of RAM, and InfiniBand nodes connectivity. Typically, 48 to 64 parallel computing cores were utilized to perform the present simulation runs.

\subsection{Limits of the present numerical framework}

The numerical model presently adopted to carry out two-phase flow simulations is based on some simplifying assumptions that limit the range of flow conditions whose flow dynamics can be reproduced in a reliable way.

The fluid specific properties are considered constant, which poses a limit on the maximum heat flux that can be applied at the wall. Under the present flow conditions ( $q$ up to $20 \mathrm{~kW} / \mathrm{m}^{2}$ ), liquid superheating never exceeds 15 K, such that no fluid properties vary by more than $10 \%$ from their saturation value when extrapolated to the supersaturated liquid region. Since the bubbles must travel downstream to the heated section of the channel before exiting, the heat flux is also bounded by the maximum bubble length that can be achieved without making the computational domain too long. This yields an upper bound on the exit vapor quality, which in the present work does not exceed $2 \%$.

The use of a computational mesh made of square cells, and the need for discretizing the liquid film by at least $7-8$ mesh cells along the radial direction, limits the ratio of the minimum film thickness to the channel diameter, and therefore the minimum mass flux that can be tested with the computational mesh adopted. Under the flow conditions of Case 1, the minimum mass flux achievable without refining the computational grid is 350 $\mathrm{kg} /\left(\mathrm{m}^{2} \mathrm{~s}\right)$, where the film thickness in this case is estimated by means of the experimental correlation of Han and Shikazono [59]. On the other hand, the 
use of a laminar flow model poses an upper limit to the mass flux in order to avoid turbulent flow, such that $G<1730 \mathrm{~kg} /\left(\mathrm{m}^{2} \mathrm{~s}\right)$ to maintain the Reynolds number below 2300 .

For a horizontal configuration, the maximum channel diameter that can be modelled by an axisymmetric geometry is about $1 \mathrm{~mm}$ according to the criterion based on the Confinement number proposed by Ong and Thome [44] $\left(\mathrm{Co}=\left(\sigma / g \Delta \rho D^{2}\right)^{1 / 2}>1\right.$ to suppress gravity effects), and hence all the channel sizes simulated here respect this condition $(\mathrm{Co}=1.45$ for the largest channel tested). Nonetheless, the pressure drop occurring in microchannels may make both the vapor density and the saturation temperature drop considerably along the streamwise direction; however, even for the smallest 0.3 mm diameter channel tested here, the total pressure drop (acceleration plus frictional) is about $0.7 \mathrm{kPa}$ which would lead to only a $0.1 \mathrm{~K}$ decrease in the saturation temperature and less than $0.1 \%$ drop in the vapor density, such that both of them are considered constant within the numerical method.

Finally, the use of values of bubble frequency and initial volume arbitrarily chosen does not reflect any real bubble generation process, which in any case may vary from one experiment to another depending on how the bubbles were produced, e.g. by boiling, by flashing [37, 38] or by a separated bubble generation chamber [60]. However, to the present authors' knowledge, a reliable model to predict the bubble generation frequency and volume within microscale flow conditions is still missing in the literature. Therefore, it was preferred not to make the present CFD results dependent on any particular model. Nevertheless, the possibility to vary the bubble frequency independently of the other flow parameters numerically allows this 
effect to be studied alone.

\section{Results}

\subsection{Validation}

The capability of the present numerical framework to simulate slug flow boiling in a microchannel for a continuous train of bubbles was demonstrated in Magnini and Thome [61]. The results from seven selected simulation runs were compared with the boiling heat transfer data of Consolini and Thome [13] in the low vapor quality region. The numerical model captured very well the experimental values of the time-averaged heat transfer coefficient for the flow conditions which are of interest here. The comparison also emphasized the essential impact of bubble frequency, whose values in the experiments were not known, on the heat transfer performance of the flow, as the heat transfer coefficient in the simulations fell by more than $50 \%$ when reducing the bubble frequency by a factor of 10 . Furthermore, in the study of the key flow parameters developed below, various benchmark comparisons between experimental and simulation data will be illustrated, thus providing various direct and indirect validations of the present numerical scheme.

\subsection{Heat flux effect}

The results of three simulation runs (Cases 1-3) where the heat flux ranges from $5 \mathrm{~kW} / \mathrm{m}^{2}$ to $20 \mathrm{~kW} / \mathrm{m}^{2}$ while keeping constant all the other flow conditions are presented here. In particular, the initial liquid slug length is fixed to $6 D$, thus giving a constant bubble frequency of $104 \mathrm{~Hz}$ which is within the range measured experimentally using a laser/diode setup by 


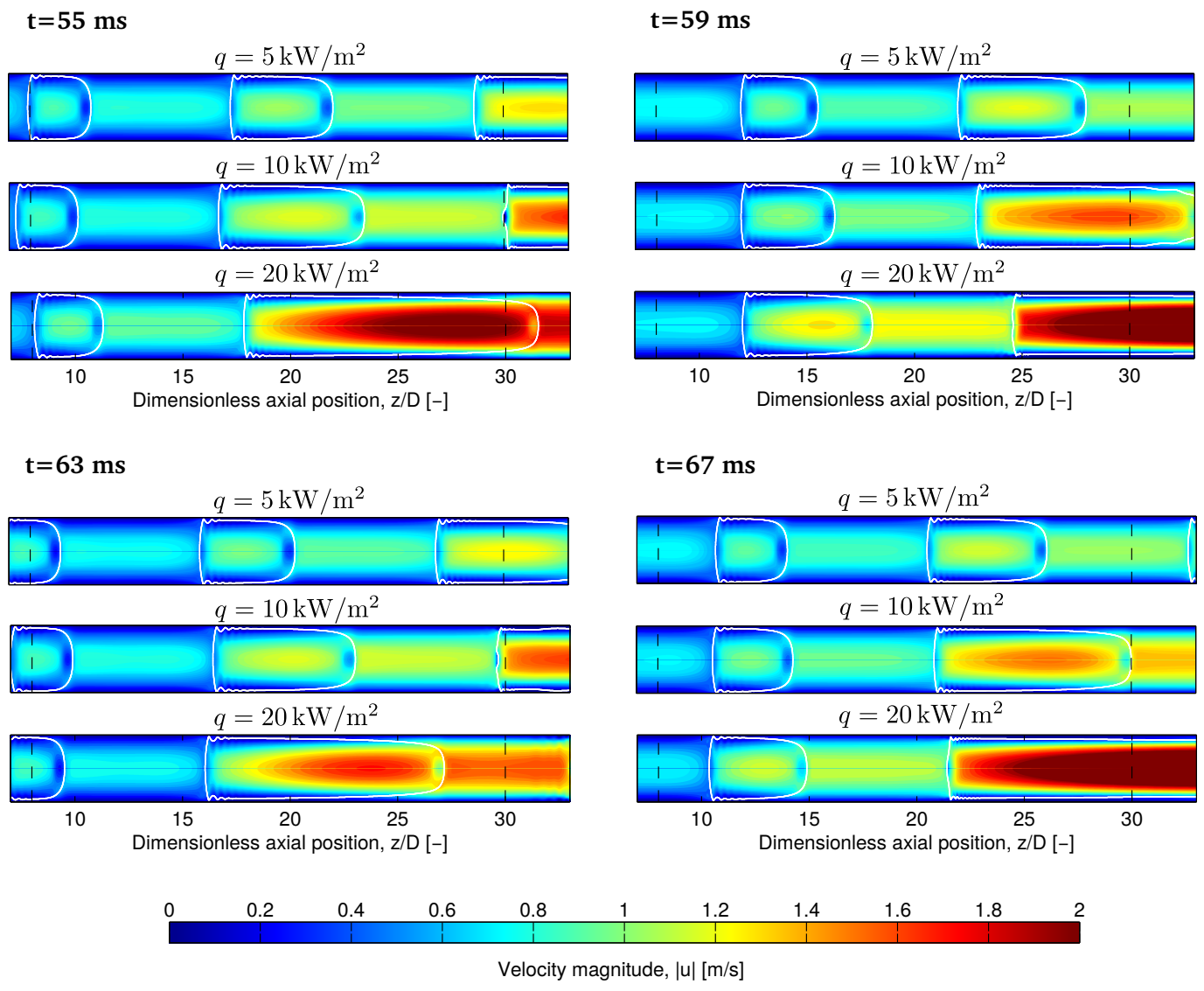

Figure 3: Bubble profiles (white solid lines) and contours of the velocity magnitude for simulations run with three different heat fluxes. The vertical black dashed lines indicate the start and end of the heated region. Figures are not to scale.

Revellin et al. [43] in a $0.5 \mathrm{~mm}$ channel. Figure 3 shows the bubble profiles and velocity contours at different time instants obtained for different values of the heat flux. It is evident that the larger heat flux induces much larger evaporation rates, which give rise to vigorous accelerations of the liquid flow in front of a bubble. The bubble which at $t=55 \mathrm{~ms}$ enters within the heated region of the channel (top-left figures), at $t=63 \mathrm{~ms}$ (bottom-left) has grown 
twice as long when comparing results for $q=20 \mathrm{~kW} / \mathrm{m}^{2}$ and $q=5 \mathrm{~kW} / \mathrm{m}^{2}$. The liquid in front of the bubble has accelerated up to about $1.5 \mathrm{~m} / \mathrm{s}$ when considering the velocity at the tube's axis for $q=20 \mathrm{~kW} / \mathrm{m}^{2}$, compared to a velocity of about $0.8 \mathrm{~m} / \mathrm{s}$ detected for the lowest heat flux condition.

Understanding the impact of the heat flux on the bubble dynamics is fundamental in order to explain the heat transfer trends. In principle, the bubble velocity grows linearly with the wall heat flux, as yielded by the theoretical model of Consolini and Thome [40] here modified as shown by Magnini et al. [24] to account for the actual liquid-vapor slip:

$$
U_{b}(z)=U_{b, 0}+\frac{4 q z}{\rho_{v} h_{l v} D}
$$

where $U_{b, 0}$ represents the adiabatic bubble velocity and $z$ denotes the streamwise coordinate. This trend is confirmed by the present CFD results. Figure 4 reports the bubble velocity versus the streamwise coordinate for three different heat fluxes for the simulations and the theoretical model. The bubble velocity is made dimensionless by the average liquid inlet velocity $U_{l}$, while the streamwise coordinate is given by taking the axial location of the entrance in the heated region, $z_{h}=8 D$, as the zero reference. The bubble velocity increases along the microchannel as an effect of evaporation, and the linear trends with $z$ and $q$ also emerge from the CFD results, which match very well the model of Consolini and Thome [40].

Accelerations have two counteracting effects on the thickness of the liquid film surrounding the bubble: the larger velocity makes the liquid film thicker, while the acceleration of the liquid ahead of the bubble results in a thinner viscous boundary layer and therefore thinner liquid film [62]. Fig- 


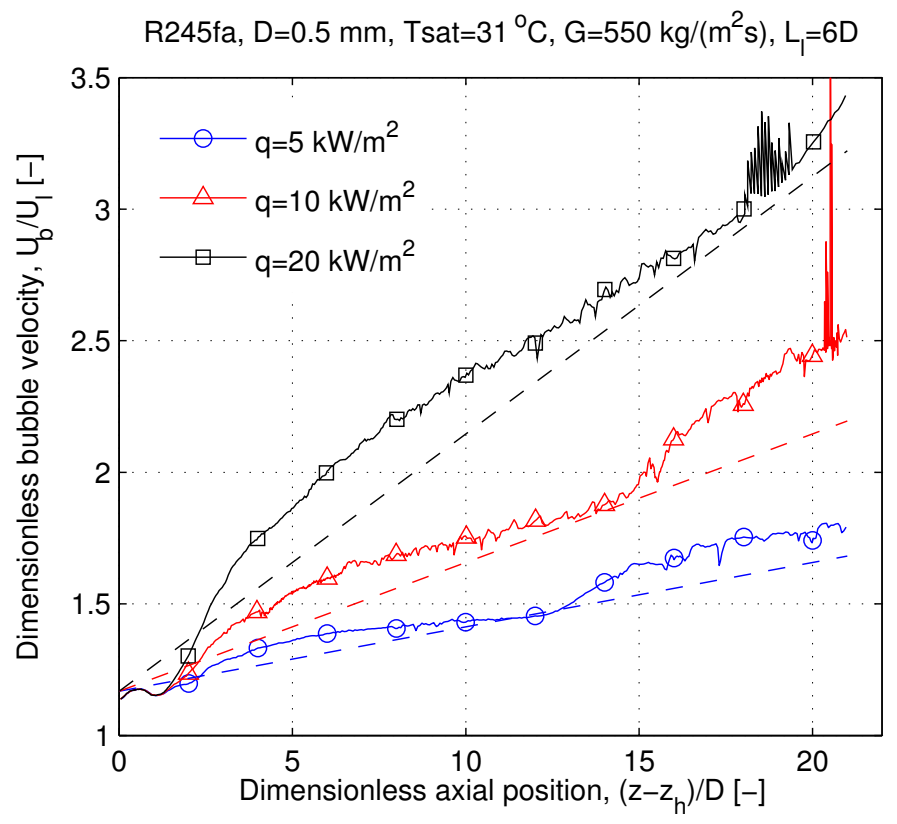

Figure 4: Bubble velocity at different values of wall heat flux. Solid lines represent CFD results, dashed lines depict results from the Consolini and Thome [40] model. $z_{h}$ identifies the entrance in the heated region.

ure 5 displays the liquid film thickness versus the streamwise coordinate obtained with the numerical simulations and those predicted by the correlation of Han and Shikazono [62] for elongated bubble flow under accelerated conditions. In the figure, the liquid film thickness is calculated as the average bubble-wall distance computed for a short region of length of $0.5 D$ downstream the wavy zone in the proximity of the rear menuscus. Note that film dryout does not occur under the simulated conditions, and therefore a thin layer of liquid always surrounds the bubble. It can be seen that under the present flow conditions, characterized by values of the bubble Capillary number $\mathrm{Ca}_{\mathrm{b}}=\mu_{l} U_{b} / \sigma$ ranging from 0.012 to 0.043 , the liquid film thickness 


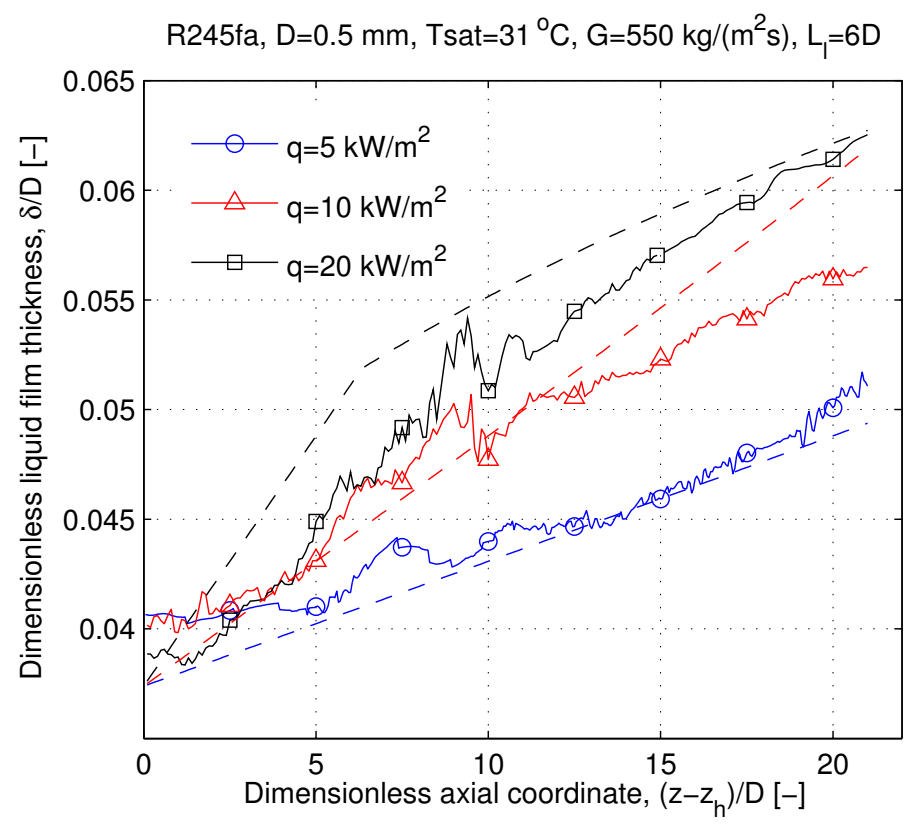

Figure 5: Liquid film thickness at different values of wall heat flux. Solid lines represent CFD results, dashed lines depict the prediction obtained from the Han and Shikazono [62] correlation. $z_{h}$ identifies the entrance in the heated region.

increases monotonically as the bubble accelerates along the microchannel, while the larger bubble velocity associated with an increase of the heat flux yields a thicker liquid film. The maximum deviation between the liquid film thickness values from the simulations and those predicted by the Han and Shikazono [62] correlation is of about $10 \%$, which is an excellent result given the range of $15 \%$ accuracy declared by the authors of the correlation. The slope change occurring in Fig. 5 for the $q=20 \mathrm{~kW} / \mathrm{m}^{2}$ predicted liquid film thickness curve at about $\left(z-z_{h}\right) / D=6$ is an effect of the Han and Shikazono [62] model, which estimates the film thickness as the minimum value given by two empirical correlations, i.e. one for steady bubble flow 
and one for accelerated flow. Mechanistically speaking, in Fig. 5 one should note that due to the relatively short vapor bubbles and low heat flux, the liquid film thinning along the microchannel resulting from film evaporation is here surpassed by the thickening effect induced by the bubble acceleration. As already demonstrated in the computational study of Magnini and Thome [61] and corroborated by the experimental observation of Borhani et al. [63], the length of the liquid slug trapped between the bubbles does not change significantly while the bubbles flow and elongate along the heated section of the microchannel, therefore the bubble frequency is uniform along the microchannel.

With regard to the heat transfer results, it was shown in [61] that the cyclic transit of elongated bubbles and liquid slugs within a microchannel causes the heat transfer coefficient to increase as time elapses, up to a steadyperiodic regime which is reached after a few bubble cycles. At this terminal stage, the heat transfer coefficient periodically grows in the liquid film region and drops in the successive liquid slug zone. Figure 6 presents the heat transfer coefficient versus time measured after 21 heated diameters during the terminal steady-periodic regime, for different values of the wall heat flux. The heat transfer coefficient is evaluated as

$$
h=\frac{q}{T_{w}-T_{s a t}}
$$

The CFD results suggest that, when the bubble frequency is maintained constant, the heat transfer coefficient decreases as heat flux increases. The time-averaged value after 21 heated diameters drops from $\bar{h}=2677 \mathrm{~W} /\left(\mathrm{m}^{2} \mathrm{~K}\right)$ when $q=5 \mathrm{~kW} / \mathrm{m}^{2}$, down to $\bar{h}=2464 \mathrm{~W} /\left(\mathrm{m}^{2} \mathrm{~K}\right)$ and $\bar{h}=2424 \mathrm{~W} /\left(\mathrm{m}^{2} \mathrm{~K}\right)$ when raising the heat flux first to $q=10 \mathrm{~kW} / \mathrm{m}^{2}$ and then to $q=20$ 


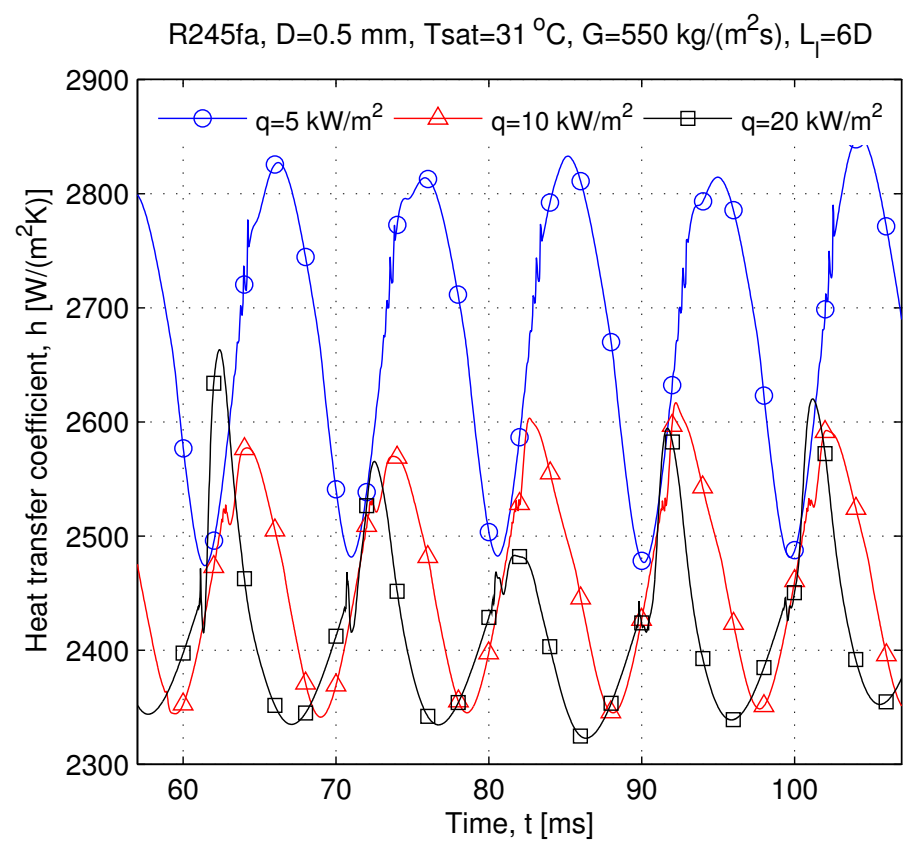

Figure 6: Time-dependent heat transfer coefficient after 21 heated diameters from the entrance in the heated section of the channel at three different values of wall heat flux. For comparison purposes, the local liquid-only single-phase value is $h_{s p}=1180 \mathrm{~W} /\left(\mathrm{m}^{2} \mathrm{~K}\right)$.

$\mathrm{kW} / \mathrm{m}^{2}$, respectively. This can be attributed to the thickening of the liquid film resulting from larger bubble velocity as is apparent in Fig. 5. The thicker liquid film is also characterized by a larger thermal inertia, which is reflected by a smaller amplitude of the temporal oscillations of the heat transfer coefficient in Fig. 6. The different heat transfer coefficient peak magnitudes for successive bubble cycles at fixed heat flux are the result of time-dependent oscillations of the rear meniscus of the bubble. These trigger capillary waves on the bubble surface which yield a minimum liquid film thickness which varies with time (this will be explained more in detail in 
Section 4.3). The magnitude of the peaks, which are always detected shortly after the passage of the bubble tail, reflects the instantaneous minimum film thickness value when the bubble tail is flowing at the axial location under analysis. The change in the peaks magnitudes at different bubble cycles becomes more evident at larger values of the heat flux, because the higher evaporation rate increases the velocity of the liquid within the slug which impinges the bubble tail at its downstream end, thus making the observed time-dependent patterns more pronounced.

When summarizing these results in a power law function of the kind $\bar{h} \sim q^{n}$, these yield:

$$
\bar{h} \sim q^{-0.07}
$$

i.e. at a fixed bubble frequency the heat transfer coefficient is a weak function of the inverse of the wall heat flux. Note that the trend emerging from the numerical simulations is not necessarily representative of experimental conditions where bubbles are generated by wall nucleation, in which an increase of the heat flux is usually associated with a larger bubble generation frequency. However, the present results are useful to isolate the effect of the wall heat flux from the other flow parameters, while the impact of the bubble frequency alone will be studied in Section 4.5.

The trend of the heat transfer coefficient versus the vapor quality can be extrapolated from the plot of Fig. 7, which represents the normalized time-averaged heat transfer coefficient along the microchannel. As the flow is far from being thermally developed (it would take about $180 \mathrm{D}$ under the simulated conditions), the dimensional value of the heat transfer coefficient decreases monotonically with $z$. Differently, the normalized heat transfer 
coefficient grows monotonically with $z$ and therefore, given that the vapor quality increases linearly with $z$ as demonstrated in [61], it increases with the vapor quality. This can be ascribed to the longer liquid film region, and to the larger liquid slug velocity which enhances the internal liquid recirculation and therefore the convection at the channel's wall. Hence, it seems reasonable to assume that, if the channel was long enough in order for the heat transfer coefficient to reach an asymptotic value, its dimensional value also would increase with the vapor quality, in agreement with the behavior indicated in Fig. 1 for the IB regime.

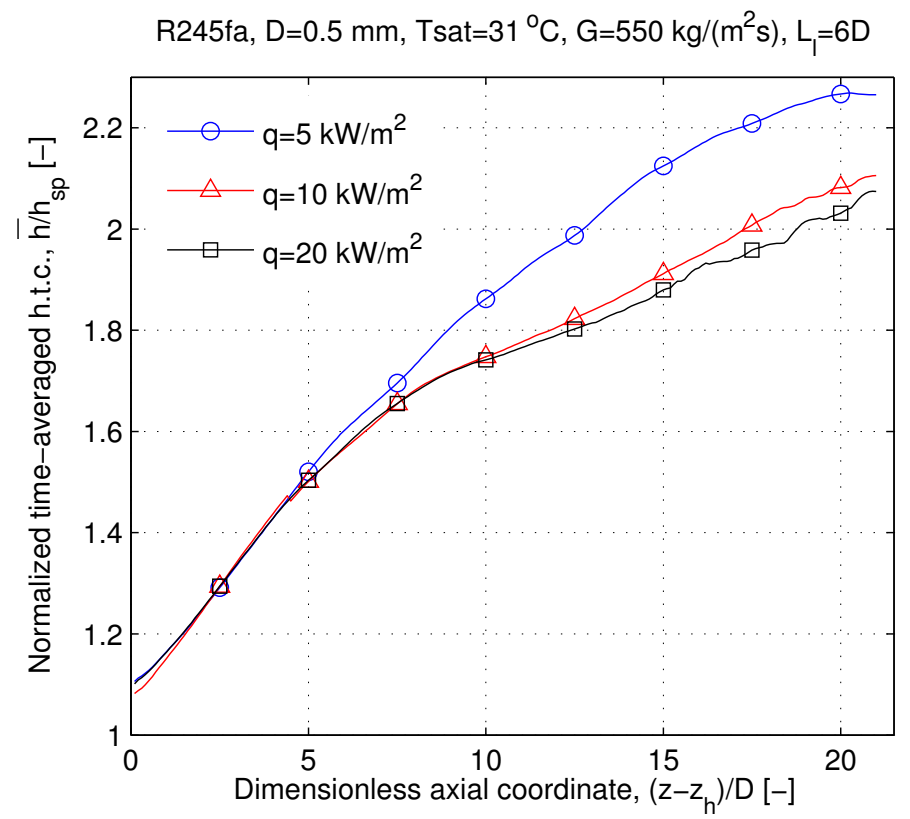

Figure 7: Time-averaged heat transfer coefficient normalized by the local liquid-only singlephase value $h_{s p}(z)$, at three different values of wall heat flux. 


\subsection{Mass flux effect}

The parametric analysis of mass flux effects is conducted by testing values of $G$ from $400 \mathrm{~kg} /\left(\mathrm{m}^{2} \mathrm{~s}\right)$ to $700 \mathrm{~kg} /\left(\mathrm{m}^{2} \mathrm{~s}\right)$, cases 1,4 , and 5 in Table 1 . The initial liquid slug length is kept constant to $L_{l}=6 D$, thus giving bubble generation frequencies which rise from $76 \mathrm{~Hz}$ at the lowest $G$ to $143 \mathrm{~Hz}$ at the highest value.

The liquid film thickness increases with the mass flux due to the larger velocity of the liquid. Figure 8 reports the values of the dimensionless liquid film thickness evaluated after 21 heated diameters, which is the location where the results for heat transfer will be presented. The diagram includes not only the average liquid film thickness, which is the mean bubble-to-wall distance in the central region of the bubble, but also shows its minimum value which is detected upon the most upstream crest of the interfacial wave occurring at the bubble surface near its tail. The minimum film thickness has to be interpreted as a time-averaged value, which is varying in time within the band reported here and which will be explained later on. The relationship between average liquid film thickness and mass flux for the present results can be expressed by the power law:

$$
\delta \sim G^{0.72}
$$

which benchmarks well with Bretherton's law [64], $\delta / D \sim \mathrm{Ca}_{\mathrm{b}}{ }^{0.67}$. The minimum film thickness also increases with the flow rate, and so does the difference between the average and minimum film thickness, $\delta-\delta_{\text {min }}$, which can be interpreted as a sort of wave amplitude. The latter's dependence on 
the flow rate is of the kind:

$$
\delta-\delta_{\min } \sim G^{0.64}
$$

which is again in good agreement with the asymptotic behavior obtained by Bretherton [64], $\delta-\delta_{\min } \sim \mathrm{Ca}_{\mathrm{b}}{ }^{0.67}$. Note that although increasing with $G$ in absolute terms, the wave amplitude remains at an almost constant fraction of the average film thickness irrespective of the flow rate, between 0.28 and 0.33 .

Within Fig. 8, the minimum film thickness is plotted with its range of temporal variation. The bubble Reynolds number $\operatorname{Re}_{\mathrm{b}}=\rho_{l} U_{b} D / \mu_{l}$, computed by referring to the actual velocity of the bubble in the simulations at the measuring point, ranges from 1000 to 1500 . At such regimes, inertia effects are known to trigger time-dependent flow effects which appear as oscillations of the bubble tail [65]. The dynamics of the interfacial wave nearby the bubble tail observed in the present simulations is influenced by such time-dependent patterns, so that the minimum film thickness oscillates in time within the range indicated in Fig. 8. The amplitude of the temporal oscillations of the minimum film thickness grows significantly with the mass flow rate due to the larger bubble Reynolds number. This may considerably reduce the gap between the most upstream wave crest and the channel's wall, in particular decrease it down to $50 \%$ less than the average film thickness value for the case with $G=700 \mathrm{~kg} /\left(\mathrm{m}^{2} \mathrm{~s}\right)$. Note that the minimum film thickness is more important than the average thickness in triggering the onset of liquid film dryout in slug flow boiling conditions.

Figure 9 depicts the time-dependent heat transfer coefficient measured after 21 heated diameters during the terminal steady-periodic regime, for 


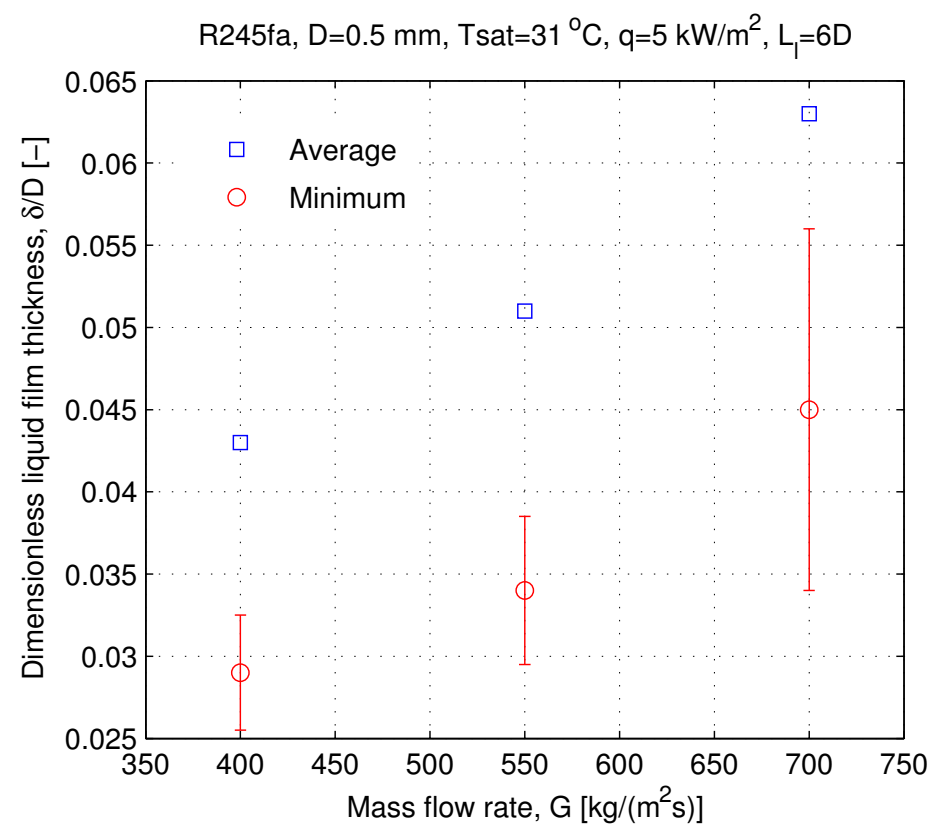

Figure 8: Liquid film thickness after 21 heated diameters at three different values of mass flux.

three different values of mass flux. As it could be expected from the analysis of the bubble dynamics above, larger values of mass flux reduce the heat transfer coefficient due to the increase of the liquid film thickness. This is qualitatively in line with microchannel heat transfer data trends, such as those of Sumith et al. [35], Agostini et al. [37], and Costa-Patry et al. [41]. The results for the $G=400 \mathrm{~kg} /\left(\mathrm{m}^{2} \mathrm{~s}\right)$ systematically show some spikes on the heat transfer coefficient at the instant in which the bubble tail is crossing the location under analysis, which are a consequence of the capillary waves at the bubble tail. The tight relationship between heat transfer and bubble interface dynamics is more evident in the inset provided with Fig. 9, where heat transfer coefficient and local liquid film thickness are plotted 


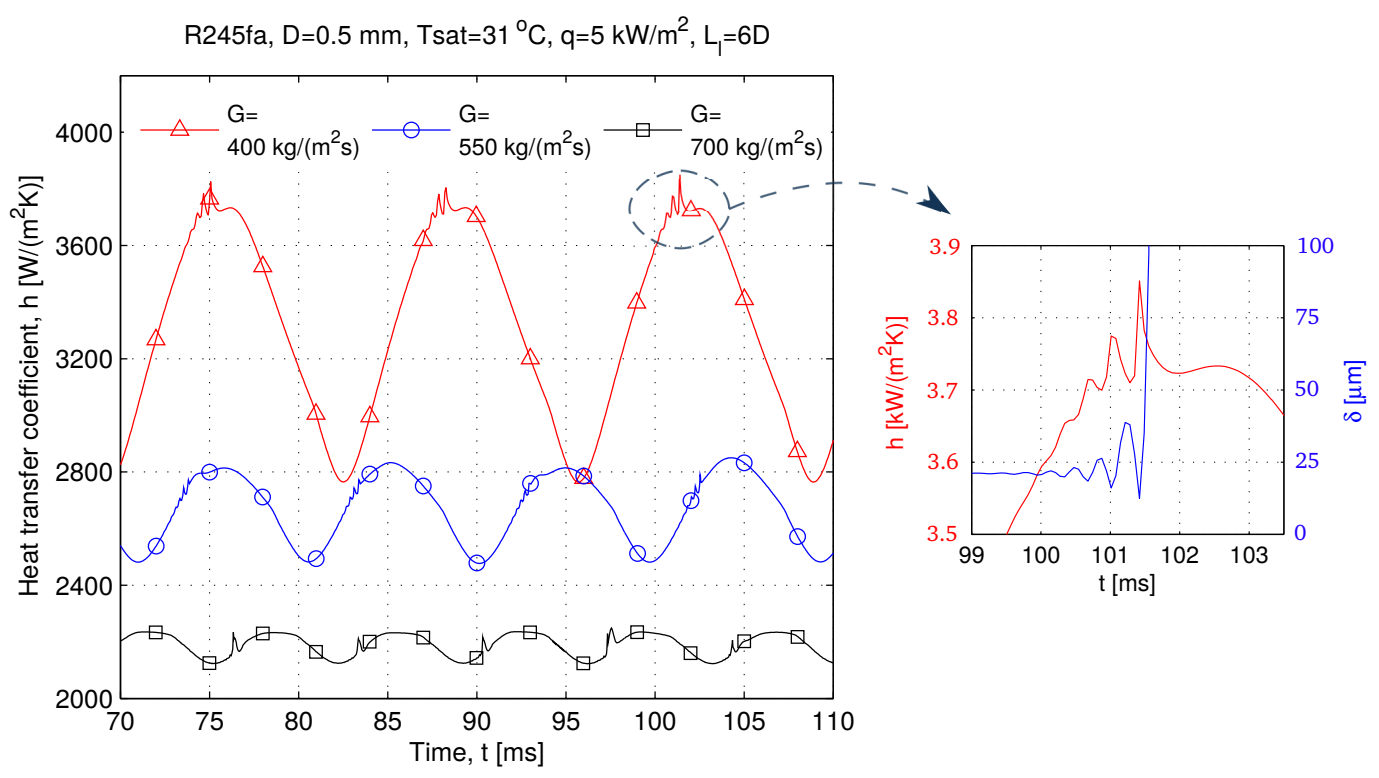

Figure 9: Time-dependent heat transfer coefficient after 21 heated diameters from the entrance in the heated section of the channel at three different values of mass flux. The small inset on the right displays in more detail the heat transfer coefficient and liquid film thickness time-dependent dynamics during the transit of the bubble tail for $G=$ $400 \mathrm{~kg} /\left(\mathrm{m}^{2} \mathrm{~s}\right)$.

together versus time $\left(G=400 \mathrm{~kg} /\left(\mathrm{m}^{2} \mathrm{~s}\right)\right)$ during the transit of the bubble rear meniscus at the axial location under study. As the liquid film gets thinner upon the interfacial wave crests, the low thermal inertia of the film for the $G=400 \mathrm{~kg} /\left(\mathrm{m}^{2} \mathrm{~s}\right)$ case makes the channel wall temperature react very fast to the change in thickness, thus inducing sharp peaks on $h$. Single spikes of variable magnitude appear on the heat transfer coefficient profile for $G=700 \mathrm{~kg} /\left(\mathrm{m}^{2} \mathrm{~s}\right)$ due to the time-dependent nature of the interfacial wave. Despite the relatively large average liquid film thickness, the significant decrease of the minimum liquid film thickness at the bubble tail for this case 
induces sharp variations of the heat transfer coefficient which are apparent in Fig. 9. As the minimum film thickness varies periodically in time, the magnitude of the spikes depends on the instantaneous amplitude of the most upstream wave crest when it passes across the location under investigation.

The relationship between the time-averaged heat transfer coefficient and mass flux emerging from the numerical results is:

$$
\bar{h} \sim G^{-0.75}
$$

with the frequency increasing by a factor of 2 within the range of $G$ considered here in order to keep a constant distance between the bubbles. The increase of the bubble frequency $f$ limits the drop of $h$ when augmenting $G$ that would otherwise be observed if $f$ was maintained constant. Note that the bubble frequency is expected to increase with the mass flux when bubbles are generated by wall nucleation [43], although a proven relationship quantifying such a dependence for microchannel flow boiling conditions is not yet available.

\subsection{Channel diameter effect}

The results of numerical simulations run with the channel diameter varied from $D=0.3 \mathrm{~mm}$ to $D=0.7 \mathrm{~mm}$ are now presented (see Table 1: runs 1, $10,11)$. In order to maintain the same distance between the bubbles $(6 D)$, the bubble frequency is changed from $104 \mathrm{~Hz}$ for $D=0.5 \mathrm{~mm}$, to $177 \mathrm{~Hz}$ for $D=0.3 \mathrm{~mm}$ and to $78 \mathrm{~Hz}$ for $D=0.7 \mathrm{~mm}$.

The liquid film thickness increases significantly with the channel diameter, not only in terms of its dimensional value, which is expected due to the larger channel, but also in its dimensionless $\delta / D$ value. As the two-phase flow is 


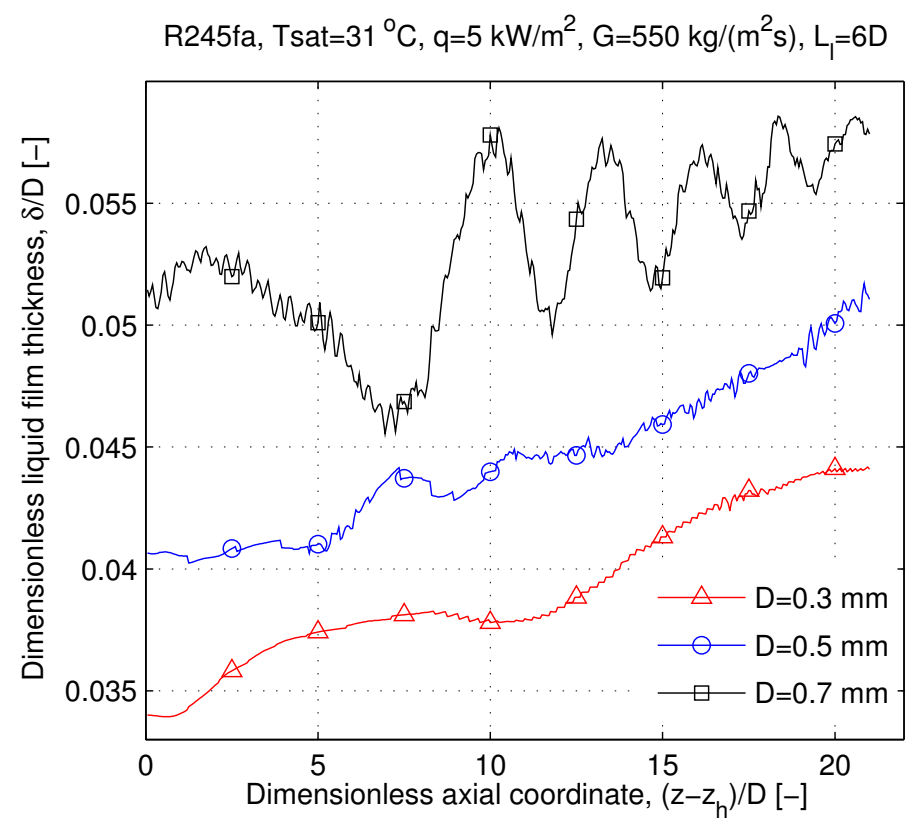

Figure 10: Liquid film thickness at different values of the channel diameter. $z_{h}$ identifies the entrance in the heated region.

well within the visco-inertial regime $(\mathrm{Re} \gg 1)$, the dimensionless liquid film thickness grows with both the Capillary number, which is independent of the channel diameter, and the Reynolds number. Figure 10 reports the liquid film thickness versus the streamwise coordinate measured along the heated section of the channel, while liquid evaporates and the bubbles grow and accelerate downstream. Considering the flow of the bubble in the initial adiabatic region of the channel, the increase of the channel diameter from $D=0.3 \mathrm{~mm}$ to $D=0.7$ yields a rise of $50 \%$ in the dimensionless liquid film thickness, as the bubble Reynolds number changes from about 500 to 1100 (while the bubble velocity is unchanged). Strong time-dependent patterns on the bubble surface are detected for the case characterized by the largest 
diameter. These trigger temporal oscillations of the liquid film thickness while the bubble is transiting within the heated section of the microchannel, as is very evident in Fig. 10. Note that the bubble velocity is not influenced by the change in the channel diameter. This is consistent with Eq. (7), because the adiabatic bubble velocity $U_{b, 0}$ is observed to change with the channel size by only $2 \%$, while the streamwise coordinate $z$ scales with the channel diameter.

The reduction of the liquid film thickness associated with the smaller channel size is known to enhance the heat transfer performance of a slug flow. This trend is confirmed by the present simulations, see Fig. 11. The

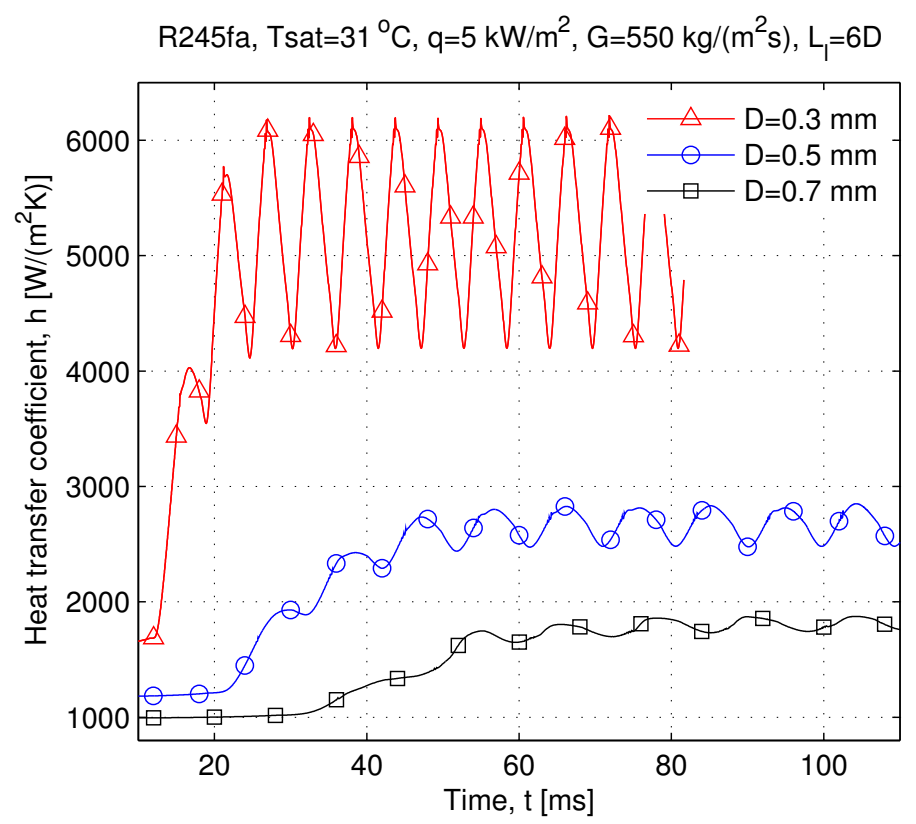

Figure 11: Time-dependent heat transfer coefficient after 21 heated diameters from the entrance in the heated section of the channel, at different values of channel diameter. 
reduced thermal inertia of the liquid film results in a faster thermal transient, as 3 bubble cycles are already sufficient to reach a terminal steady-periodic stage for the wall heat transfer when $D=0.3 \mathrm{~mm}$, but also in larger and sharper temporal variations of the heat transfer coefficient during the cyclic transit of bubble and liquid slug pairs. Since the liquid film thickness scales more than proportionally with the channel diameter, the time-averaged heat transfer coefficient depends on the channel diameter as:

$$
\bar{h} \sim D^{-1.24}
$$

Making the film thickness explicit, an almost linear relationship between heat transfer coefficient and the inverse of the liquid film thickness is derived for the present results:

$$
\bar{h} \sim \frac{1}{\delta^{0.94}}
$$

which would be expected for a one-dimensional steady-state heat conduction across the film. This is in agreement with the three-zone boiling heat transfer model of Thome et al. [17] for slug flow in microchannels, although the model did not include the effect of thermal inertia on the heat transfer across the liquid film.

A better insight on the transient dynamics governing heat transfer at the different channel diameters is possible by rescaling time and heat transfer coefficient as it is done in Fig. 12. Time is made dimensionless by the pair (bubble and liquid slug) transit period $\tau=1 / f$, and $t / \tau=0$ corresponds to the transit of the nose of the seventh generated bubble at the axial location under analysis. The heat transfer coefficient is expressed by the Nusselt number, $\mathrm{Nu}=h D / \lambda_{l}$. The time-averaged Nusselt number still increases 


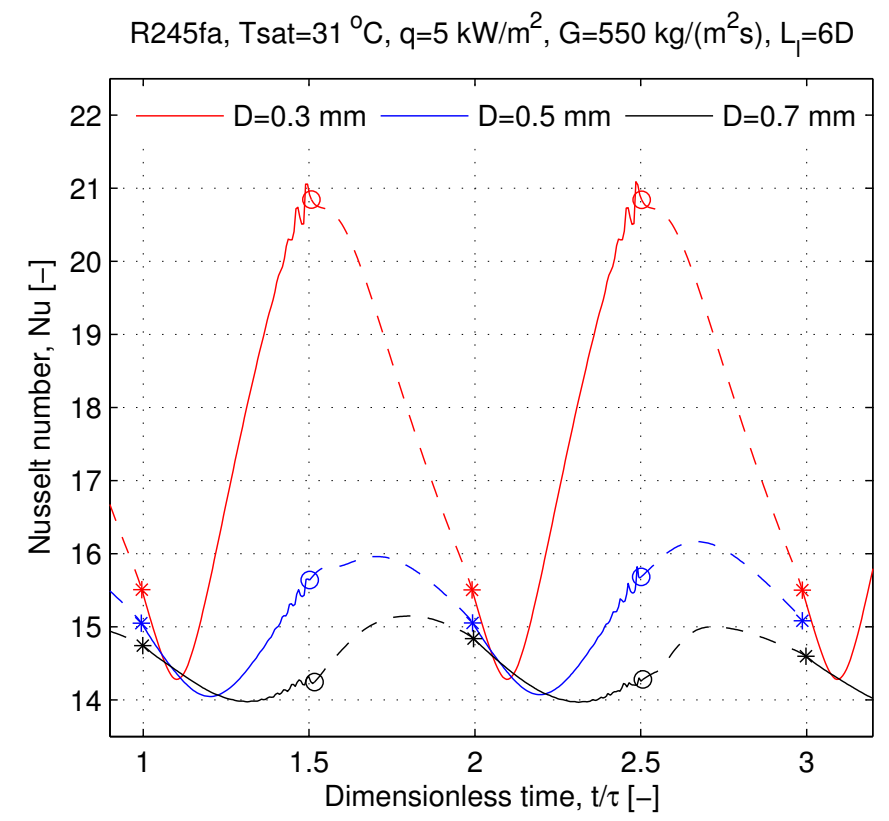

Figure 12: Time-dependent Nusselt number after 21 heated diameters from the entrance in the heated section of the channel at different values of channel diameter. Segments depicted by solid lines identify the transit of the vapor bubble (liquid film), dashed lines identify the liquid slug. Circles mark the passage of the bubble tail, asterisks locate the bubble nose.

when reducing the channel size, which derives from the dependence of the heat transfer coefficient on the channel diameter indicated in Eq. (13). The minimum Nusselt number magnitude is similar for all the values of the channel diameter tested (about $\mathrm{Nu}=14$ ), and it is always measured while the liquid film zone is transiting. The time interval between the bubble nose passage and the detection of the minimum Nusselt number, which can be interpreted as the time it takes for heat conduction to become the governing heat transfer mechanism in the film zone, depends on the shape of the 
bubble nose and the thermal inertia of the liquid film. An increase of the channel size increases the thermal inertia of the film, and at the same time the larger inertial effect on the bubble motion makes its nose more slender, thus retarding the formation of a thin liquid film. This results in a larger time delay for heat conduction to become effective in the liquid film region. It is observed that, in average, the minimum value of the Nusselt number is measured at about $1.5 D$ upstream from the bubble nose for $D=0.3 \mathrm{~mm}$ while at $3 D$ and $4.3 D$ upstream from the bubble nose for $D=0.5 \mathrm{~mm}$ and $D=0.7 \mathrm{~mm}$, respectively.

The maximum magnitude of the Nusselt number increases as the channel size is reduced as an effect of the more effective heat conduction across the liquid film. This maximum is located in the film region (exactly at the bubble tail) for $D=0.3 \mathrm{~mm}$, while it moves to the liquid slug region for bigger channels. This emphasizes a change in the relative importance of the mechanisms governing heat transfer in the different flow regions. For smaller channels, the heat transfer performance drops as soon as the bubble tail has passed, thus suggesting that heat convection within the liquid slug is significantly less effective than heat conduction in the liquid film. As the channel size is increased, the location where the maximum heat transfer performance is observed shifts within the liquid slug zone. On average, the maximum Nusselt number is identified when the bubble tail is $2.2 D$ and $2.83 D$ downstream the axial location under analysis for $D=0.5 \mathrm{~mm}$ and $D=$ $0.7 \mathrm{~mm}$, respectively. This suggests an enhanced effect of heat convection from the recirculating flows within the liquid slug over heat conduction within the liquid film. Note that, in the present simulations, at the measuring point 
(after 21 heated diameters) the vapor bubble and liquid slug have almost the same length.

In summary, under the present operating conditions, radial heat conduction across the film seems to dominate heat transfer for channel sizes below $D=0.3 \mathrm{~mm}$, while heat convection within the liquid slug seems to prevail at larger diameters, with the positive effect of radial heat conduction across the film gradually vanishing at channel diameters greater than $0.7 \mathrm{~mm}$.

\subsection{Bubble generation frequency effect}

The effect of the bubble generation frequency on bubble dynamics and heat transfer is analyzed by performing simulations with different initial lengths of the liquid slug trapped between the bubbles, from a minimum of $L_{l}=0.5 \mathrm{D}$ to a maximum of $L_{l}=10 \mathrm{D}$, while keeping constant all the other flow parameters (see Table 1: cases 1,6-9). This corresponds to values of the bubble generation frequency ranging from $72 \mathrm{~Hz}$ to $269 \mathrm{~Hz}$. The initial bubble length is set to the same value for all the simulated cases, $L_{v}=3 D$.

As the distance among the flowing bubbles is reduced, the time available for the thermal boundary layer at the wall to develop after the transit of a leading bubble decreases. The trailing bubble grows absorbing the sensible heat from the superheated liquid in the thermal boundary layer ahead of it, and hence the less energy stored by the liquid due to a shorter distance among the bubbles yields a lower evaporation rate. Figure 13 represents the length of the vapor bubble as it flows within the heated and the successive terminal adiabatic sections of the microchannel, at different values of the liquid slug length. As anticipated above, the initial bubble length is the same for all 


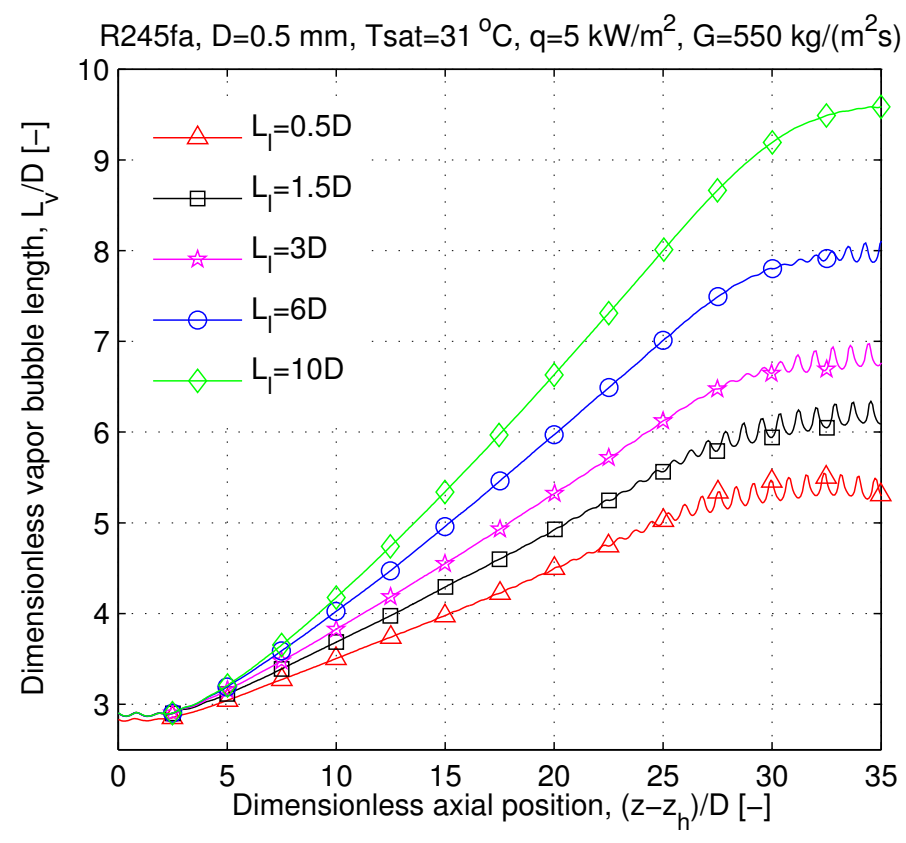

Figure 13: Bubble lengths at different values of the liquid slug length (i.e. bubble frequency). $z_{h}$ identifies the entrance in the heated region. $\left(z-z_{h}\right) / D=22$ is the end of the heated region, followed by a terminal adiabatic zone.

the test runs $\left(L_{v} \approx 3 D\right)$, then the smaller evaporation rate characterizing the higher bubble frequency cases is reflected by bubbles which grow less in length. At the axial location where the time-dependent heat transfer coefficient is usually reported in the present work, $\left(z-z_{h}\right) / D=21$, the bubble length reduces from about $7 D$ to $4.5 D$ within the range of bubble frequencies tested here. It is worth to note that although the evaporation rate of the single bubble decreases as the bubble frequency is increased, the larger interfacial area of the overall two-phase flow consequent to the augmented bubble count enhances the efficiency of the evaporation process. Comparing the change in vapor quality extracted from the computational 
results to its theoretical maximum value derived from an energy balance ( $\Delta x=4 q L_{h} /\left(G D h_{l v}\right)$ where $L_{h}$ is the heated length), the fraction of the wall heat flux turned into latent heat grows from $76 \%$ when $L_{l}=10 D$ to $98.6 \%$ when $L_{l}=0.5 \mathrm{D}$. Obviously, in the last case there is no possibility of nucleate boiling being a factor here as claimed in many Chen [66] type microchannel flow boiling correlations.

Note that, as already indicated in Section 4.2, the liquid slug length and bubble frequency for each simulated case do not change significantly from their initial values as the flow develops along the microchannel.

In the cases treated in the previous sections, the thickness of the liquid film trapped between the bubble and the wall during evaporation was generally a function of the velocity of the bubble. This emerges also from the Bretherton's [64] and Taylor's [67] laws and the most recent work from Han and Shikazono [59, 62], where the liquid film thickness is a function of the Capillary and Reynolds numbers only. Under the flow conditions studied in this section, the bubble velocity versus streamwise coordinate curves for different liquid slug lengths, which are not reported here, do not show any appreciable differences, i.e. their velocity plots overlap. Figure 14 displays the liquid film thickness versus streamwise coordinate for different liquid slug lengths. The liquid film thickness exhibits different values, in particular for the case with $L_{l}=0.5 D$, which cannot be ascribed to different bubble velocities. For $L_{l}>0.5 D$, a specific trend is identified. All the cases are characterized by the same liquid film thickness in the initial adiabatic region of the channel. Then, as evaporation begins, the liquid film thickening rate is less as the bubble frequency is reduced. These different trends can 


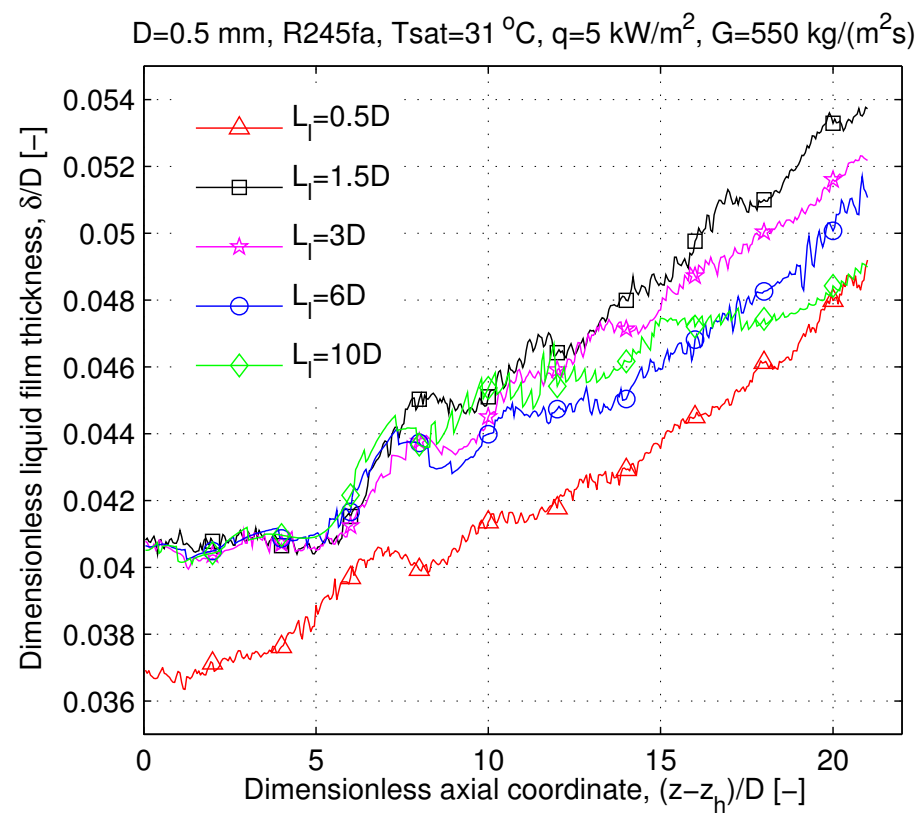

Figure 14: Liquid film thickness at different values of the liquid slug length (i.e. bubble frequency). $z_{h}$ identifies the entrance in the heated region.

be attributed to the different length of the bubble under the different flow conditions, as observed in Fig. 13. Khodaparast et al. [65] pointed out that in the visco-inertial flow regime, which is of interest here, when interfacial waves appear at the tail of the bubble, the liquid film thickness decreases gradually from the nose of the bubble to the wavy region at the bubble tail. As a consequence, the longer the bubble is and the thinner the liquid film becomes, until an asymptotic condition is reached when the bubble is several diameters long. Therefore, if an average liquid film thickness is calculated as it is done here, this value depends not only on the bubble velocity, but also on the bubble length, which is a very peculiar effect of the visco-inertial regime. 
The same effect arises in the present study. A lower bubble frequency, which yields longer bubbles without changing significantly their velocity, gives a thinner liquid film as is evident from Fig. 14.

The case run with the shortest liquid slug length, $L_{l}=0.5 D$, exhibits a liquid film thickness versus streamwise coordinate curve which stays below the others. Already the value of the adiabatic liquid film thickness, $\delta / D=$ 0.037, is smaller than the thickness measured for the cases run with $L_{l}>$ $0.5 D, \delta / D=0.041$. This comes from bubble proximity effects, i.e. the mutual interaction among the bubbles. As emphasized by Aussillous and Quéré [68], the development of the viscous boundary layer at the rear of a leading bubble poses an upper limit to the liquid film thickness of the trailing one. If the viscous boundary layer of the liquid flow field ahead of the nose of the bubble is thicker than the liquid film that would occur for an isolated bubble, the usual laws for predicting the liquid film thickness (such as Bretherton's [64] and Taylor's [67] law) are still valid. Otherwise, the actual liquid film thickness would be lower compared to the isolated bubble case, and Aussillous and Quéré [68] demonstrated by means of experiments that it would match the viscous boundary layer thickness $\delta_{v b l}$, evaluated as:

$$
\frac{\delta_{v b l}}{D}=\frac{1}{D}\left(\frac{\mu_{l} L_{l}}{\rho_{l} U_{b}}\right)^{1 / 2}=\left(\frac{L_{l}}{D} \frac{1}{\mathrm{Re}_{\mathrm{b}}}\right)^{1 / 2}
$$

Under the present flow conditions, Eq. (15) suggests that, for $L_{l}<1.4 D$, the viscous boundary layer becomes thinner than the liquid film measured around bubbles which do not interact with each other $(\delta / D=0.041)$. Therefore, when the liquid slug length is smaller than $1.4 D$, the actual liquid film thickness is expected to be smaller than the value measured in case of longer liquid slugs, thus motivating and explaining the different trend emerging for 
the $L_{l}=0.5 D$ case in Fig. 14 .

The heat transfer coefficient decays in the liquid slug zone, and therefore the shorter residence time of the liquid slug corresponding to a larger bubble frequency is expected to enhance the heat transfer performance. This trend is respected by the present CFD simulations, as it emerges from Fig. 15 which presents the time-dependent heat transfer coefficient at different values of liquid slug length. The shorter distance among the bubbles yields a smaller amplitude of the temporal oscillations of the heat transfer coefficient. Except for the $L_{l}=0.5 \mathrm{D}$ flow configuration, where the bubble proximity effects make the liquid film thinner and thus further augmenting the heat transfer

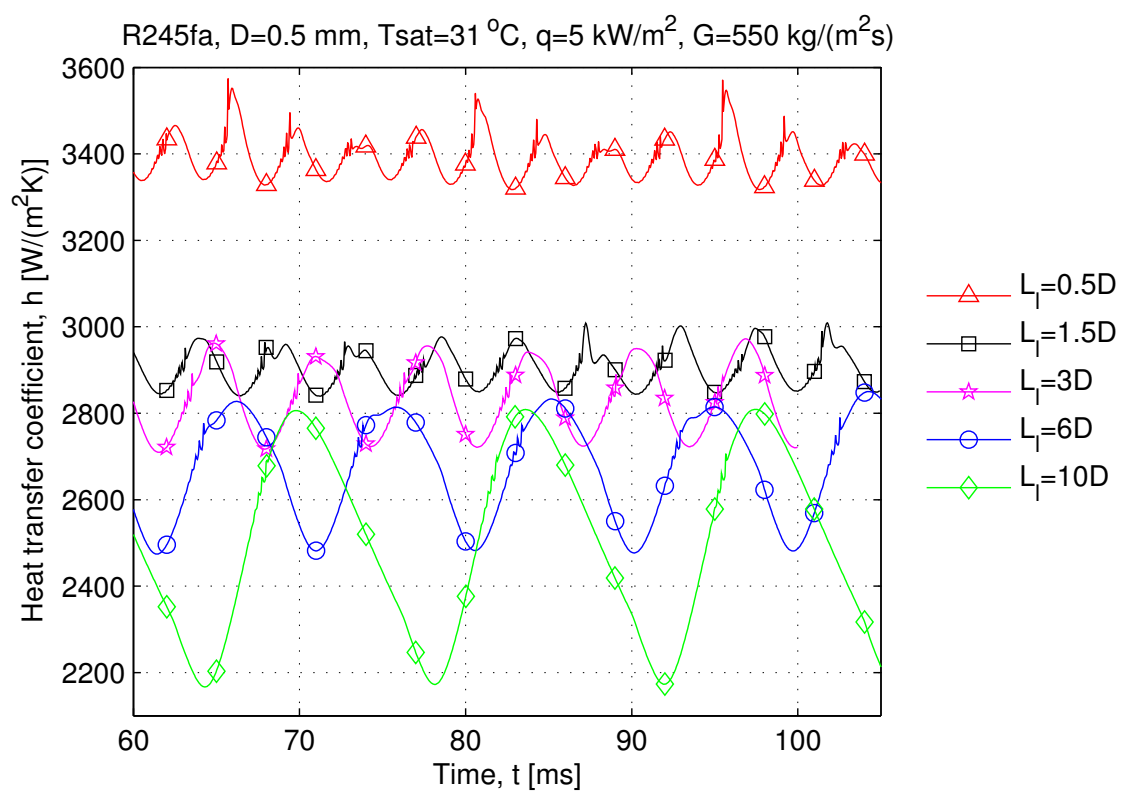

Figure 15: Time-dependent heat transfer coefficient after 21 heated diameters from the entrance in the heated section of the channel, at different values of liquid slug length (i.e. bubble frequency). 
performance, the other cases show comparable behaviors. The maximum magnitude of the heat transfer coefficient is similar for all the cases (between $2800 \mathrm{~W} /\left(\mathrm{m}^{2} \mathrm{~K}\right)$ and $\left.3000 \mathrm{~W} /\left(\mathrm{m}^{2} \mathrm{~K}\right)\right)$, while its minimum magnitude decreases as the liquid slug length increases, such that the time-averaged heat transfer coefficient rises with the bubble frequency.

Excluding the range of liquid slug lengths where bubble proximity effects become important, the relationship between the time-averaged heat transfer coefficient and the liquid slug length arising from this study is:

$$
\frac{\bar{h}-\bar{h}_{s p}}{\bar{h}_{s p}} \sim\left(\frac{L_{l}}{D}\right)^{-0.133}
$$

Note that usual correlations for laminar developing flow yield a heat transfer coefficient which decays with the square root of the inverse of the distance from the point where heating commences. The smaller exponent extracted from the present numerical results indicates a slower decay with the liquid slug length, which is an effect of the recirculating flows acting within the liquid slug which provide fresh liquid from the channel's axis to the wall. The corresponding trend of the time-averaged heat transfer coefficient with the bubble generation frequency is:

$$
\bar{h} \sim f^{0.22}
$$

with the exponent 0.22 rising to 0.59 when considering the $L_{l}=0.5 D$ and $1.5 \mathrm{D}$ cases only. If the bubble frequency could be expressed as an increasing function of the heat flux, $f \sim q^{n}$ with $n>0$ as in Dupont et al. [46], then Eq. (17) would indicate that the heat transfer coefficient augments with the heat flux due to the increased bubble frequency, i.e. not due to nucleate boiling as claimed in many experimental papers. In particular, when taking 
$n=1.74$ as in [46], Eq. (17) yields $\bar{h} \sim q^{0.38}$ if the entire range of bubble frequencies analyzed in this section is considered, and $\bar{h} \sim q^{1.02}$ when limiting the analysis to $L_{l}=0.5 D$ and $1.5 D$ only. This can explain the $\bar{h} \sim q^{0.7}$ dependency reported in many microchannel flow boiling studies and often attributed to a nucleate boiling effect.

\subsection{Saturation temperature effect}

The saturation temperature of the fluid is varied from $10^{\circ} \mathrm{C}$ to $50^{\circ} \mathrm{C}$ (test runs $1,12,13)$ and the effect of the different fluid properties on the bubble dynamics under evaporation and boiling heat transfer coefficient is studied. All the other parameters are unchanged, and their values are reported in Table 1. As the saturation temperature is increased, thus bringing the fluid toward the critical condition, liquid properties such as density and viscosity decrease and the related vapor properties increase in value, while surface tension and vaporization latent heat fall. In particular, within the range of saturation temperatures analyzed here, the liquid density decreases by $8 \%$ while the vapor density rises by a factor of 4 , such that the liquid to vapor density ratio drops from 281 at $T_{\text {sat }}=10^{\circ} \mathrm{C}$ to 66 at $T_{\text {sat }}=50^{\circ} \mathrm{C}$. The liquid viscosity diminishes by $40 \%$ with a consequent increase of the Reynolds number of the flow. The latent heat and surface tension drop off respectively by $12 \%$ and $32 \%$. Since all the fluid properties are to some extent influenced by the change in saturation temperature, its effect on the slug flow boiling heat transfer is indeed very complex.

Concerning the adiabatic bubble dynamics, the Capillary number of the flow is almost unchanged when increasing the saturation temperature (viscosity and surface tension decrease by a similar percentage), but the signifi- 


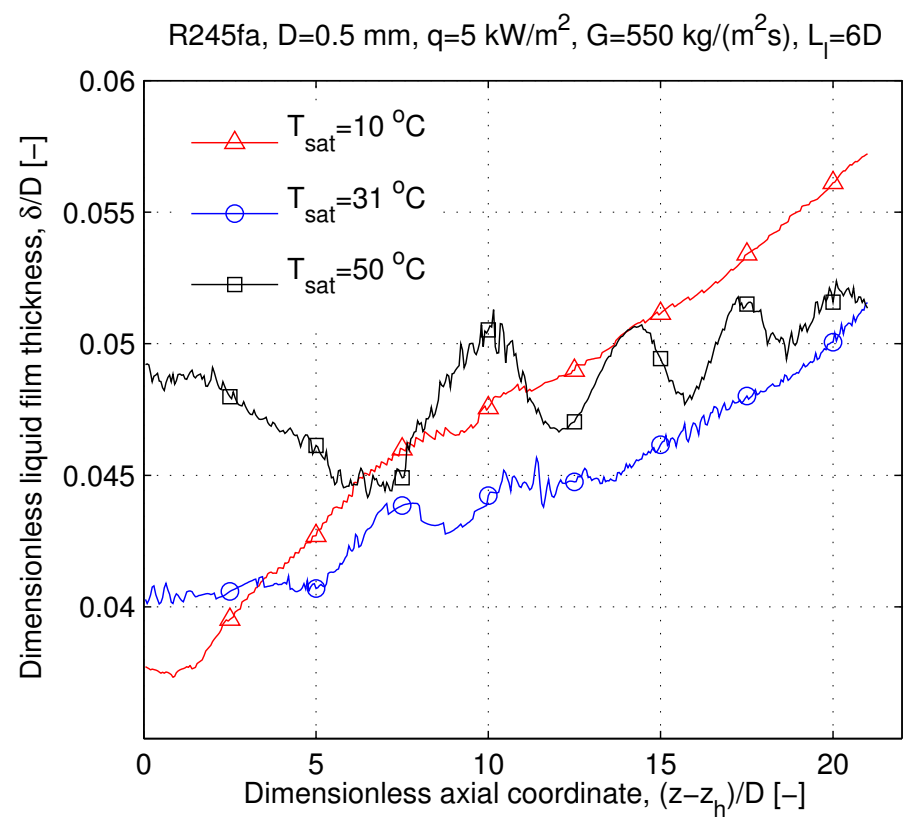

Figure 16: Liquid film thickness at different values of saturation temperature. $z_{h}$ identifies the entrance in the heated region.

cant increment of the Reynolds number makes inertial effects on the bubble dynamics more apparent and the liquid film substantially thicker, as demonstrated by Fig. 16. The adiabatic bubble velocity is only slightly affected by the change in the saturation temperature (mostly via the liquid density, as $\left.U_{l}=G / \rho_{l}\right)$. However, as evaporation occurs, the large liquid to vapor density ratio corresponding to a lower saturation temperature results in a great expansion of the liquid turning into vapor, which is reflected by dramatic bubble accelerations, see Fig. 17 which depicts the bubble velocity along the microchannel at the different saturation temperatures. Such a large increase of the bubble velocity at low saturation temperatures modifies the liquid film thickness trends reported in Fig. 16, as the flow condition for which 
R245fa, $D=0.5 \mathrm{~mm}, \mathrm{q}=5 \mathrm{~kW} / \mathrm{m}^{2}, \mathrm{G}=550 \mathrm{~kg} /\left(\mathrm{m}^{2} \mathrm{~s}\right), \mathrm{L}_{1}=6 \mathrm{D}$

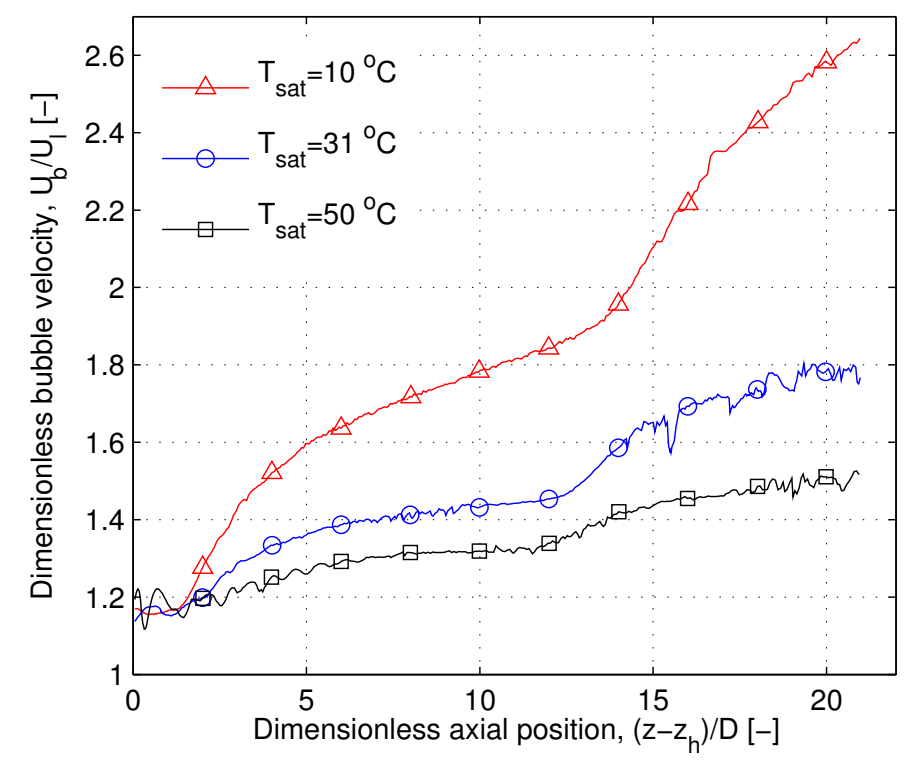

Figure 17: Bubble velocity at different values of saturation temperature. $z_{h}$ identifies the entrance in the heated region.

$T_{\text {sat }}=10^{\circ} \mathrm{C}$ gives the thickest liquid film when considering cross-sections towards the end of the heated section of the microchannel.

Fig. 18 shows the time-dependent heat transfer coefficient measured after 21 heated diameters. Despite the highest liquid film thickness, the case with the lowest saturation temperature gives the largest heat transfer coefficient. This is due to the shorter liquid slug residence time, $36 \%$ of the pair period against $56 \%$ for the $T_{\text {sat }}=50^{\circ} \mathrm{C}$ case, deriving from the much longer vapor bubble, which limits the drop of the heat transfer coefficient in the liquid slug zone. Furthermore, the higher Péclet number of the flow, $\mathrm{Pe}=U_{b} D / \alpha_{t, l}$ with $\alpha_{t, l}$ being the liquid thermal diffusivity, enhances heat convection within the liquid slug. The time-averaged heat transfer coefficient for the intermediate 


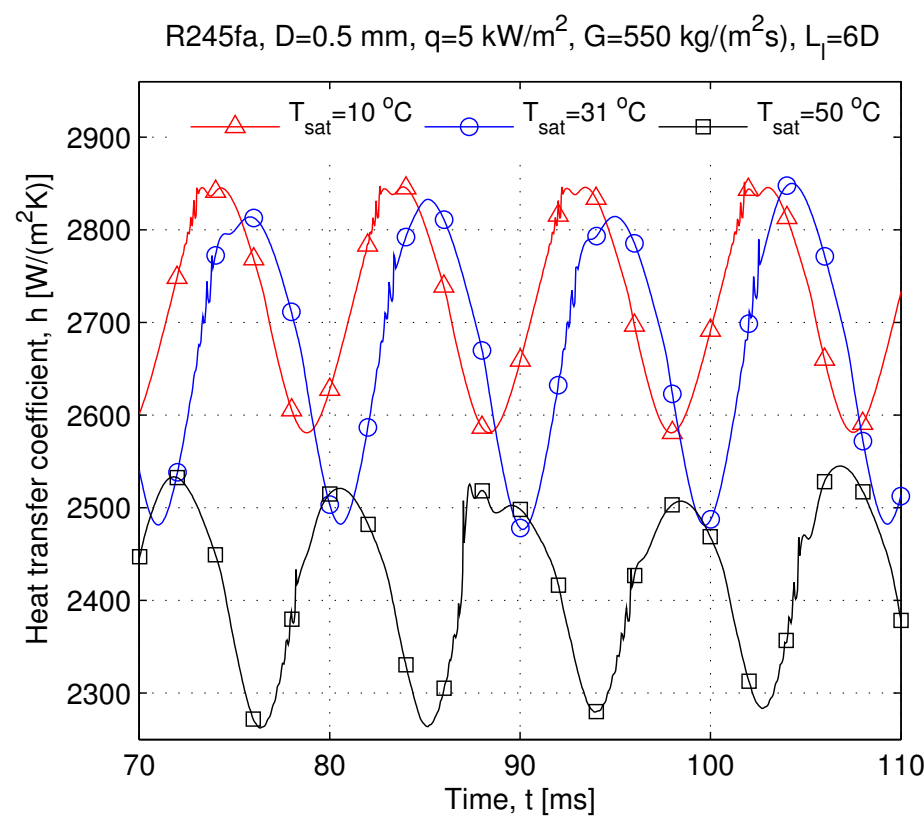

Figure 18: Time-dependent heat transfer coefficient after 21 heated diameters from the entrance in the heated section of the channel, at different values of saturation temperature.

case with $T_{\text {sat }}=31{ }^{\circ} \mathrm{C}$ is only slightly smaller than the lowest saturation temperature case, as the negative effect of the longer liquid slug residence time (about $50 \%$ of the pair period) is partially balanced by the thinner liquid film. The time-averaged heat transfer coefficient at the highest saturation temperature tested is about $12 \%$ smaller compared to the value measured at $T_{\text {sat }}=10^{\circ} \mathrm{C}$, mainly because of the longer residence time of the liquid slug zone.

In summary, the present CFD results indicate that the heat transfer performance falls when increasing the saturation temperature. This, however, does not account for the increase in the expected bubble frequency with increasing pressure that would occur if bubbles were generated by nucleate 
boiling [46], which would have an opposite effect. Therefore, the dependence of slug flow boiling heat transfer on the saturation temperature is rather complex. The saturation temperature influences numerous parameters such as liquid film thickness, bubble velocity, growth rate, residence times, etc., with trends which are far from being linear or even monotonic. For this reason, at present no attempt is made to derive a simplified relationship between the numerical results and saturation temperature.

\section{Discussion}

In this section, a dimensional analysis of the parameters influencing bubble dynamics and heat transfer for microchannel slug flow boiling is developed. Boiling heat transfer in microchannel two-phase flow has been for a long time investigated regardless of the actual geometric configuration of the liquid-vapor interface, so that the dimensionless groups usually adopted did not consider the real two-phase flow structure. However, the first studies on the motion of bubbles within capillaries can be dated back to 1935 [69], and numerous works tackling many different aspects of the bubble dynamics have been published ever since. Hence, the objective of this section is to merge available models for bubble dynamics and heat transfer in order to identify the dimensionless groups actually governing boiling heat transfer in the peculiar slug flow configuration, in conjunction with the results of the CFD simulations presented in Section 4. The analysis is restricted to the flow conditions of interest here, i.e. the low vapor quality region where no dryout occurs, so that the slug flow can be interpreted as a sequential transit of a vapor bubble surrounded by a liquid film, and a liquid slug. 


\subsection{Dimensionless parameters governing liquid film thickness}

The parametric analysis conducted in Section 4 emphasized the leading role of the liquid film thickness in determining the heat transfer performance of slug flow. In particular, the effect of heat flux, mass flux and channel diameter could all be explained by referring to the change in the liquid film size. Therefore, it is useful to first identify the dimensionless groups upon which its thickness depends. Han and Shikazono [62] showed that the liquid film thickness for accelerating bubbles could be expressed as a function of the Capillary, Reynolds and Bond acceleration numbers of the bubble:

$$
\delta=D \cdot \mathrm{f}_{1}\left(\mathrm{Ca}_{\mathrm{b}}, \mathrm{Re}_{\mathrm{b}}, \mathrm{Bd}_{\mathrm{b}}\right)
$$

to be evaluated at the actual velocity and acceleration of the bubble, here denoted respectively as $U_{b}$ and $a_{b}$, as follows:

$$
\mathrm{Ca}_{\mathrm{b}}=\frac{\mu_{l} U_{b}}{\sigma}, \quad \operatorname{Re}_{\mathrm{b}}=\frac{\rho_{l} U_{b} D}{\mu_{l}}, \quad \mathrm{Bd}_{\mathrm{b}}=\frac{\rho_{l} a_{b} D^{2}}{\sigma}
$$

Note that Eq. (18) is a simplified model which does not include the bubble length, which was however observed in Section 4.5 to slightly influence the liquid film thickness in the inertial regime of the bubble flow, and the liquid slug length, which may limit the development of the viscous boundary layer and therefore the film thickness. However, these are here considered to be secondary effects on the bubble dynamics and therefore they are discarded from the present analysis. The bubble velocity at a given streamwise location $z$ within the microchannel can be obtained by Eq. (7), here rewritten to highlight the dimensionless groups involved:

$$
U_{b}=U_{b, 0}+4 U_{l} \operatorname{Bo}\left(\frac{\rho_{l}}{\rho_{v}}\right)\left(\frac{z}{D}\right)
$$


where $\mathrm{Bo}=q /\left(G h_{l v}\right)$ is identified as the Boiling number, $U_{l}=G / \rho_{l}$ denotes the average liquid velocity at the inlet, and $z=0$ denotes the entrance in the heated region. The bubble velocity under adiabatic conditions $U_{b, 0}$ depends on the adiabatic liquid film thickness $\delta_{0}$ and liquid velocity:

$$
U_{b, 0}=\frac{U_{l}}{1-4 \delta_{0} / D\left(1-\delta_{0} / D\right)}=c\left(\delta_{0} / D\right) U_{l}
$$

where $c$ is an explicit function of the initial liquid film thickness. Therefore, the bubble velocity can be expressed as:

$$
U_{b}=U_{l}\left[c\left(\frac{\delta_{0}}{D}\right)+4 \operatorname{Bo}\left(\frac{\rho_{l}}{\rho_{v}}\right)\left(\frac{z}{D}\right)\right]
$$

The adiabatic liquid film thickness is known to depend on the bubble Capillary and Reynolds numbers [59] evaluated at the adiabatic bubble velocity:

$$
\delta_{0}=D \cdot \mathrm{f}_{2}\left(\mathrm{Ca}_{\mathrm{b}, 0}, \mathrm{Re}_{\mathrm{b}, 0}\right)
$$

Equations (21) and (23) have to be solved together in a coupled fashion starting from the Capillary and Reynolds numbers evaluated at the average velocity of the liquid, so that finally the converged values of adiabatic liquid film thickness and bubble velocity only depend on the liquid Capillary and Reynolds numbers, $\mathrm{Ca}_{1}=\mu_{l} U_{l} / \sigma$ and $\mathrm{Re}_{1}=\rho_{l} U_{l} D / \mu_{l}$. Hence, the bubble velocity takes the following form:

$$
U_{b}=U_{l}\left[c\left(\mathrm{Ca}_{1}, \mathrm{Re}_{\mathrm{l}}\right)+4 \mathrm{Bo}\left(\frac{\rho_{l}}{\rho_{v}}\right)\left(\frac{z}{D}\right)\right]
$$

The bubble acceleration is calculated from the parameters already identified above as follows:

$$
a_{b}=\frac{d U_{b}}{d t}=U_{b} \frac{d U_{b}}{d z}=4 \frac{U_{b} U_{l}}{D}\left(\frac{\rho_{l}}{\rho_{v}}\right) \text { Bo }
$$


and, substituting Eq. (25) in the expression for the acceleration Bond number appearing in Eq. (19):

$$
\mathrm{Bd}_{\mathrm{b}}=4 \mathrm{Ca}_{\mathrm{b}} \operatorname{Re}_{\mathrm{l}} \operatorname{Bo}\left(\frac{\rho_{l}}{\rho_{v}}\right)
$$

In conclusion, all the dimensionless parameters appearing in Eq. (18) can be rewritten in terms of five dimensionless numbers which only involve input of flow parameters, such that the liquid film thickness is fully determined by the following dimensionless groups:

$$
\delta=D \cdot \mathrm{f}_{3}\left(\mathrm{Ca}_{\mathrm{l}}, \mathrm{Re}_{\mathrm{l}}, \mathrm{Bo}, \frac{\rho_{l}}{\rho_{v}}, \frac{z}{D}\right)
$$

that are explained as follows:

- The liquid Capillary number, $\mathrm{Ca}_{1}=\mu_{l} U_{l} / \sigma$, compares viscous to surface tension forces, and the liquid film becomes thicker with increasing $\mathrm{Ca}_{1}$. The Capillary number is proportional to the mass flow rate and therefore a larger mass flow rate tends to make the liquid film thicker, as observed in Section 4.3.

- The liquid Reynolds number, $\operatorname{Re}_{1}=\rho_{l} U_{l} D / \mu_{l}$, compares inertial to viscosity effects on the bubble. The Reynolds number is responsible for the appearance of an interfacial wave on the bubble surface and, in the range of flow conditions studied here, the liquid film thickness grows with $\mathrm{Re}_{1}$. Larger channel diameters or mass fluxes contribute to increase the liquid film thickness, even in its dimensionless $\delta / D$ form.

- The Boiling number, Bo $=q /\left(G h_{l v}\right)$ can be interpreted as the ratio between the evaporation mass flux $q / h_{l v}$ and the bulk mass flux. The 
bubble acceleration resulting from liquid evaporation is proportional to the Boiling number, and therefore the liquid film gets thicker with increasing Bo.

- The liquid to vapor density ratio, $\rho_{l} / \rho_{v}$, determines the rate of expansion of the liquid when it turns into vapor because of phase change, and hence the bubble acceleration. Higher saturation temperatures reduce the density ratio of the fluid and, as a consequence, the bubble acceleration, but the overall effect on the liquid film thickness also depends on the relative variation of the Reynolds number, as inferred in Section 4.6.

- The liquid film thickens with the dimensionless distance, $z / D$, travelled by the bubble within the heated section of the channel, due to the acceleration effect.

\subsection{Dimensionless parameters governing heat transfer}

In microchannel slug flow, boiling heat transfer can be modelled separately for the zones into which the flow can be decomposed. In absence of film dryout, these are the liquid film (vapor bubble) and liquid slug zones. In the liquid film region, heat transfer has been successfully modelled by assuming one-dimensional radial heat conduction across the stagnant liquid layer $[17,19]$. Note that the stagnant film approximation becomes less valid as the Capillary and Reynolds number of the flow are increased, due to the appearance of non-negligible axial velocity components within the film [65]. The nondimensional problem governing heat transfer across the liquid film 
can be formulated as follows:

$$
\begin{aligned}
& \frac{\partial T^{*}}{\partial t^{*}}=\frac{\partial^{2} T^{*}}{\partial y^{*^{2}}}, \quad t^{*}>0,0<y^{*}<1 \\
& \left.\frac{\partial T^{*}}{\partial y^{*}}\right|_{y^{*}=0}=-1 \\
& T^{*}\left(y^{*}=1\right)=T_{\text {sat }}^{*} \\
& T^{*}\left(y^{*}, t^{*}=0\right)=F\left(y^{*}\right)
\end{aligned}
$$

where the dimensionless variables are derived from:

$$
T^{*}=\frac{\lambda_{l}}{q \delta} T, \quad y^{*}=\frac{y}{\delta}, \quad t^{*}=\frac{\alpha_{t, l}}{\delta^{2}} t=\mathrm{Fo}
$$

$F\left(y^{*}\right)$ is the initial condition for the temperature which, at the terminal steady-periodic regime, is identified by the temperature profile at the end of the previous liquid slug zone. Note that the liquid film thickness is chosen as a length scale, such that the spatial coordinate ranges from 0 (at the channel's wall) to 1 (at the bubble interface), while Fo is the Fourier number and it represents the nondimensional time. The thermal problem defined by Eq. (28), with the boundary conditions given in Eqs. (29), and (30), and the initial condition given by Eq. (31), has an analytical solution which was presented in [24] and yields the following expression for the time-dependent heat transfer coefficient in the vapor bubble region:

$$
h_{v}=\frac{\lambda_{l}}{\delta} \frac{1}{T_{w}^{*}-T_{s a t}^{*}}=\frac{\lambda_{l}}{\delta} \frac{1}{1+\sum_{m=1}^{N} c_{m, v} Y_{m, v}\left(y^{*}=0\right) \mathrm{e}^{-\beta_{m, v}^{2} \text { Fo }}}
$$

where $\beta_{m, v}$ and $Y_{m, v}$ represent respectively the $m-t h$ eigenvalue and eigenfunction of the spatial solution in the film region and $c_{m, v}$ is a constant which accounts for the initial temperature profile. 
In the liquid slug region, Magnini et al. [25] demonstrated that the presence of a recirculating flow slows down the decay of the heat transfer coefficient compared to a single-phase thermal developing region. They modelled heat transfer by assuming one-dimensional radial heat conduction across a stagnant layer of liquid bounded between the channel wall and the core region of the liquid slug where vortices occurred. The related nondimensional thermal problem can be written as:

$$
\begin{aligned}
& \frac{\partial T^{*}}{\partial t^{*}}=\frac{\partial^{2} T^{*}}{\partial y^{*^{2}}}, \quad t^{*}>0,0<y^{*}<1 \\
& \left.\frac{\partial T^{*}}{\partial y^{*}}\right|_{y^{*}=0}=-1 \\
& \left.\frac{\partial T^{*}}{\partial y^{*}}\right|_{y^{*}=1}=-\operatorname{Bi}\left[T^{*}\left(y^{*}=1\right)-T_{s}^{*}\right] \\
& T^{*}\left(y^{*}, t^{*}=0\right)=G\left(y^{*}\right)
\end{aligned}
$$

where the thickness of the wall adherent layer is assumed to be the same as the liquid film thickness, so that the dimensionless variables are defined as in Eq. (32). $G\left(y^{*}\right)$ is the initial temperature profile and it is determined by the solution at the end of the previous liquid film region. $T_{s}$ is a reference temperature for the liquid slug. $\mathrm{Bi}=h_{s} \delta / \lambda_{l}$ identifies the Biot number, and $h_{s}$ is the heat transfer coefficient at the fictitious boundary (dividing streamline) between the wall adherent and recirculating flow regions within the liquid slug. This can be expressed as [70]:

$$
h_{s}=h_{s}\left(\mathrm{Pe}, L_{l} / D\right)
$$

where $\mathrm{Pe}$ is the Péclet number of the flow, $\mathrm{Pe}=U_{b} D / \alpha_{t}$. The solution of the thermal problem in the slug region yields an analytical relationship for 
the time-dependent heat transfer coefficient in the liquid slug region which takes the following form:

$$
h_{l}=\frac{\lambda_{l}}{\delta} \frac{1}{1+\sum_{m=1}^{N} c_{m, l} Y_{m, l}\left(y^{*}=0\right) \mathrm{e}^{-\beta_{m, l}^{2} \mathrm{Fo}}+\frac{1}{\mathrm{Bi}}+4 \frac{\delta}{D} \mathrm{FO}}
$$

The time-averaged heat transfer coefficient of the bubble and liquid slug pair is:

$$
\bar{h}=\frac{t_{v}}{\tau} \overline{h_{v}}+\frac{t_{l}}{\tau} \overline{h_{l}}
$$

where $t_{v}$ and $t_{l}$ are the bubble and liquid slug residence times, respectively. By assuming that the liquid slug and vapor bubble travel at the same velocity, the fraction of the pair period occupied by each zone only depends on the length of each region:

$$
\frac{t_{v}}{\tau}=\frac{L_{v}}{L_{v}+L_{l}}, \quad \frac{t_{l}}{\tau}=\frac{L_{l}}{L_{v}+L_{l}}
$$

Building on the analysis developed above, the time-averaged heat transfer coefficient for the slug flow can be related to the following parameters:

$$
\bar{h}=\frac{\lambda_{l}}{\delta} \cdot \mathrm{f}_{4}\left(\mathrm{Bi}, \mathrm{Fo}, \frac{L_{v}}{D}, \frac{L_{l}}{D}\right)
$$

which implies that the heat transfer performance depends on the five dimensionless groups which fully determine the liquid film thickness given by Eq. (27), plus four groups which govern the heat transfer process:

- The Biot number, $\mathrm{Bi}=h_{s} \delta / \lambda_{l}$, is an index of the conductive resistance across the wall adherent liquid film in the liquid slug compared to the convective resistance between the wall adherent film and the bulk flow. The larger the Biot number, the more efficient becomes the 
convection of fresh fluid from the liquid slug bulk region to the channel wall, thus enhancing the thermal performance of the liquid slug zone. This effect is promoted by shorter liquid slugs (or a high bubble frequency) and a large Péclet number of the flow.

- The Fourier number, Fo $=\alpha_{t, l} t / \delta^{2}$, characterizes the thermal performance of the slug flow in the transient regime, and it is an indicator of the thermal inertia of the liquid film and wall adherent liquid layer in the liquid slug region. The trend of the average heat transfer coefficient with the Fourier number is rather complex, as it influences the heat transfer in the liquid film and the slug regions in opposite ways. A larger Fourier number, i.e. reduced thermal inertia, enhances the heat transfer coefficient in the liquid film. On the other hand, it also shortens the reaction time of the heat transfer coefficient to the change of boundary conditions from the liquid film to the liquid slug zone, thus yielding a faster and steeper drop of the heat transfer performance in the liquid slug region as it is noticeable in Fig. 12 for the case $D=0.3$ $\mathrm{mm}$. Therefore, the overall effect of the Fourier number depends on the relative size of the vapor bubble and liquid slug zones.

- The vapor bubble and liquid slug dimensionless lengths, $L_{v} / D$ and $L_{l} / D$, determine the residence time of the two zones. Since the heat transfer coefficient increases in the liquid film region as time elapses, as long as film dryout does not occur the time-averaged heat transfer coefficient grows with the vapor bubble length. Conversely, a longer liquid slug, where the heat exchange performance falls with time, reduces the 
thermal efficiency of the slug flow.

Based on the dimensional and analytical analysis above and the simulations in Section 4, many of the numerous hydrodynamic and thermal characteristics of flow boiling in microchannels have been illustrated and explained. The next future step in this work will be to bring these mechanisms and models into an updated three-zone model.

\section{Conclusions}

A CFD analysis of the parameters influencing heat transfer in saturated flow boiling within circular microchannels was performed in the present study. A slug flow at low vapor quality condition which is characteristic of the isolated bubble flow regime where bubbles are still relatively short and liquid film dryout does not occur, was modelled. Within the numerical model, a continuous stream of bubbles was generated by prescribing the initial bubble volume and frequency, and an adiabatic entrance length was used to allow for flow development. This allowed the flow boiling process to be studied regardless of the bubble generation phenomenon, so that the bubble frequency could be varied independently of the other flow parameters. The effect of vapor quality, heat flux, mass flux, channel diameter, bubble frequency, and saturation temperature on the slug flow boiling heat transfer coefficient timeaveraged and time-dependent magnitude and trends were investigated, in order to clarify the very diverse trends emerging from the literature review conducted at the beginning of this paper. The peculiar flow structure of a slug flow strongly influences the local and average heat transfer performance via the liquid film thickness, bubble length and velocity, and therefore in the 
present work their influence on heat transfer was always studied in conjunction with the induced bubble dynamics. The major outcomes of this work can be summarized as follows:

1. The heat transfer coefficient slightly decreases with an increase of the heat flux when the bubble frequency is constant, because the liquid film becomes thicker as an effect of the augmented acceleration of the bubble.

2. An increase of the mass flow rate reduces the heat transfer performance due to the thicker liquid film. The bubble flow is usually in the visco-inertial regime, and therefore a larger Reynolds number triggers time-dependent patterns (capillary waves) on the bubble surface near the tail of the bubble which are identified as local spikes on the time-varying heat transfer coefficient. The time-dependent amplitude of these interfacial waves may locally reduce the film thickness to $50 \%$ of its average value.

3. The heat transfer coefficient augments when reducing the channel diameter, showing a $\bar{h} \sim 1 / \delta$ dependence. The thermal inertia of the flow influences significantly the response time of the channel wall to a change of the transiting zone (liquid film or liquid slug), and the location where the maximum and minimum values of the time-dependent heat transfer coefficient are measured.

4. A higher bubble frequency enhances heat transfer, thanks to the shorter residence time of the liquid slug zone. This yields a heat transfer dependence on heat flux similar to that reported in many microchannel flow boiling studies and often attributed to a nucleate boiling effect. 
Secondary effects on the bubble dynamics, such as film thickness dependence on the bubble length, and bubble proximity effects limiting the liquid film extension for very short liquid slugs, become apparent when studying a wide range of liquid slug lengths.

5. The effect of the saturation temperature is very complex due to the opposite variations induced on the fluid properties. For a constant bubble frequency, the prevalent effect is a reduction of the heat transfer coefficient for higher saturation temperatures as an effect of the smaller liquid to vapor density ratio and therefore shorter bubbles, such that the residence time of the liquid slug becomes relatively longer.

6. A dimensional analysis showed that slug flow boiling heat transfer in microchannels mainly depends on 9 dimensionless groups: the liquid Capillary and Reynolds numbers, the Boiling number, the density ratio and the dimensionless length of the channel, which govern the bubble dynamics; the Biot and Fourier numbers and the dimensionless vapor bubble and liquid slug lengths, which determine the heat transfer performance. The effects and trends created by the flow parameters investigated in the present study can all be explained by referring to the dimensionless groups derived.

\section{Acknowledgements}

M. Magnini is supported by the Swiss National Science Foundation (SNSF) under Contract No. 200020-156181. 


\section{Nomenclature}

\section{Roman Letters}

a acceleration $\left[\mathrm{m} / \mathrm{s}^{2}\right]$

$\mathrm{Bd} \quad$ acceleration Bond number, $\rho a D^{2} / \sigma[-]$

Bi Biot number, $h \delta / \lambda[-]$

Bo Boiling number, $q /\left(G h_{l v}\right)[-]$

Ca Capillary number, $\mu U / \sigma[-]$

Co Confinement number, $\sqrt{\sigma /\left(g \Delta \rho D^{2}\right)}[-]$

c constant

$c_{p} \quad$ constant pressure specific heat $[\mathrm{J} /(\mathrm{kg} \cdot \mathrm{K})]$

$D \quad$ diameter $[\mathrm{m}]$

Fo Fourier number, $\alpha_{t} t / \delta^{2}[-]$

$\boldsymbol{F}_{\boldsymbol{\sigma}} \quad$ surface tension force vector per unit volume $\left[\mathrm{N} / \mathrm{m}^{3}\right]$

$f \quad$ bubble frequency $[\mathrm{Hz}]$

$G \quad \operatorname{mass}$ flux $\left[\mathrm{kg} /\left(\mathrm{m}^{2} \cdot \mathrm{s}\right)\right]$

$\boldsymbol{g}$ gravity acceleration vector $\left[\mathrm{m} / \mathrm{s}^{2}\right]$

$h \quad$ heat transfer coefficient $\left[\mathrm{W} /\left(\mathrm{m}^{2} \cdot \mathrm{K}\right)\right]$

$h_{s} \quad$ heat transfer coefficient at dividing streamline $\left[\mathrm{W} /\left(\mathrm{m}^{2} \cdot \mathrm{K}\right)\right]$

$h_{l v} \quad$ vaporization latent heat $[\mathrm{J} / \mathrm{kg}]$

L length $[\mathrm{m}]$

$M \quad$ molecular weight $[\mathrm{kg} / \mathrm{mol}]$

$\mathrm{Nu} \quad$ Nusselt number, $h D / \lambda[-]$

$\dot{m} \quad$ interphase mass transfer $\left[\mathrm{kg} /\left(\mathrm{m}^{2} \cdot \mathrm{s}\right)\right]$

Pe Péclet number, $U D / \alpha_{t}[-]$

$p \quad$ pressure $[\mathrm{Pa}]$ 
$q \quad$ heat flux $\left[\mathrm{W} / \mathrm{m}^{2}\right]$

Re Reynolds number, $\rho U D / \mu[-]$

$R_{g} \quad$ universal gas constant $[\mathrm{J} /(\mathrm{mol} \cdot \mathrm{K})]$

$T$ temperature $[\mathrm{K}]$

$T_{s} \quad$ liquid slug reference temperature $[\mathrm{K}]$

$t$ time $[\mathrm{s}]$

$U \quad$ velocity $[\mathrm{m} / \mathrm{s}]$

$\boldsymbol{u}$ velocity vector $[\mathrm{m} / \mathrm{s}]$

$x \quad$ vapor quality $[-]$

$Y \quad$ eigenfunction $[-]$

$y, z \quad$ vertical and streamwise coordinate $[\mathrm{m}]$

$z_{h} \quad$ axial location of the entrance in the heated region $[\mathrm{m}]$

\section{Greek Letters}

$\alpha \quad$ volume fraction $[-]$

$\alpha_{t} \quad$ thermal diffusivity $\left[\mathrm{m}^{2} / \mathrm{s}\right]$

$\beta \quad$ eigenvalue $[1 / \mathrm{m}]$

$\gamma \quad$ accommodation coefficient $[-]$

$\delta \quad$ liquid film thickness $[\mathrm{m}]$

$\kappa \quad$ interface curvature $[1 / \mathrm{m}]$

$\lambda \quad$ thermal conductivity $[\mathrm{W} /(\mathrm{m} \cdot \mathrm{K})]$

$\mu \quad$ dynamic viscosity $[\mathrm{Pa} \cdot \mathrm{s}]$

$\rho \quad$ density $\left[\mathrm{kg} / \mathrm{m}^{3}\right]$

$\sigma \quad$ surface tension coefficient $[\mathrm{N} / \mathrm{m}]$

$\tau \quad$ pair (bubble and liquid slug) transit period [s] 


\begin{tabular}{ll}
\multicolumn{2}{l}{ Subscripts } \\
0 & adiabatic conditions \\
$b$ & bubble \\
$l$ & liquid \\
$s a t$ & saturation \\
$s p$ & single phase \\
$v$ & vapor \\
$w$ & wall
\end{tabular}

\section{References}

[1] B. Agostini, M. Fabbri, J. E. Park, L. Wojtan, J. R. Thome, B. Michel, State of the art of high heat flux cooling technologies, Heat Transfer Engineering 28 (2007) 258-281.

[2] J. R. Thome, State-of-the-art overview of boiling and two-phase flows in microchannels, Heat Transfer Engineering 27 (2006) 4-19.

[3] T. Saenen, M. Baelmans, Numerical model of a two-phase microchannel heat sink electronics cooling system, Int. J. of Thermal Sciences 59 (2012) 214-223.

[4] J. Braz Marcinichen, D. Wu, S. Paredes, J. R. Thome, B. Michel, Dynamic flow control and performance comparison of different concepts of two-phase on-chip cooling cycles, Applied Energy 114 (2014) 179-191.

[5] M. Kreutzer, F. Kapteijn, J. Moulijn, C. Kleijn, J. Heiszwolf, Inertial and interfacial effects on pressure drop of Taylor flow in capillaries, AIChE Journal 51 (2005) 2428-2440. 
[6] S. Waelchli, P. R. von Rohr, Two-phase flow characteristics in gas-liquid microreactors, Int. J. of Multiphase Flow 32 (2006) 791-806.

[7] M. Kashid, D. Agar, Hydrodynamics of liquid-liquid slug flow capillary microreactor: Flow regimes, slug size and pressure drop, Chemical Engineering Journal 131 (2007) 1-13.

[8] B. Agostini, J. R. Thome, Comparison of an extended database for flow boiling heat transfer coefficients in multi-microchannels elements with the three-zone model, in: Proc. of ECI International Conference on Heat Transfer and Fluid Flow in Microscale, Castelvecchio Pascoli, Italy, 2005.

[9] C. B. Tibiriçá, G. Ribatski, Flow boiling in micro-scale channels Synthesized literature review, Int. J. of Refrigeration 36 (2013) 301324.

[10] C. Baldassari, M. Marengo, Flow boiling in microchannels and microgravity, Progress in Energy and Combustion Science 39 (2013) 1-36.

[11] Y. Wang, Z. Wang, An overview of liquid-vapor phase change, flow and heat transfer in mini- and micro-channels, Int. J. of Thermal Sciences 86 (2014) 227-245.

[12] S. Lin, P. A. Kew, K. Cornwell, Flow boiling of refrigerant R141B in small tubes, Chemical Engineering Research and Design 79 (2001) $417-424$.

[13] L. Consolini, J. R. Thome, Micro-channel flwo boiling heat transfer of R-134a, R-236fa, and R-245fa, Microfluid Nanofluid 6 (2009) 731-746. 
[14] S. Szczukiewicz, N. Borhani, J. R. Thome, Two-phase heat transfer and high-speed visualization of refrigerant flows in $100 \mathrm{x} 100 \mu \mathrm{m}^{2}$ silicon multi-microchannels, Int. J. of Refrigeration 36 (2013) 402-413.

[15] S. Szczukiewicz, M. Magnini, J. R. Thome, Proposed models, ongoing experiments, and latest numerical simulations of microchannel twophase flow boiling, Int. J. of Multiphase Flow 59 (2014) 84-101.

[16] W. Qu, I. Mudawar, Flow boiling heat transfer in two-phase microchannel heat sinks - II. Annular two-phase flow model, Int. J. of Heat and Mass Transfer 46 (2003) 2773-2784.

[17] J. R. Thome, V. Dupont, A. M. Jacobi, Heat transfer model for evaporation in microchannels. Part I: presentation of the model, Int. J. of Heat and Mass Transfer 47 (2004) 3375-3385.

[18] A. Cioncolini, J. R. Thome, Algebraic turbulence modeling in adiabatic and evaporating annular two-phase flow, Int. J. of Heat and Fluid Flow 32 (2011) 805-817.

[19] T. Harirchian, S. V. Garimella, Flow regime-based modeling of heat transfer and pressure drop in microchannel flow boiling., Int. J. of Heat and Mass Transfer 55 (2012) 1246-1260.

[20] A. Mukherjee, S. G. Kandlikar, Z. J. Edel, Numerical study of bubble growth and wall heat transfer during flow boiling in a microchannel, Int. J. of Heat and Mass Transfer 54 (2011) 3702-3718.

[21] C. Fang, M. David, A. Rogacs, K. Goodson, Volume of fluid simulation 
of boiling two-phase flow in a vapor-venting microchannel, Frontiers in Heat and Mass Transfer 1 (2010) 013002.

[22] R. Zhuan, W. Wang, Flow pattern of boiling in micro-channel by numerical simulation, Int. J. of Heat and Mass Transfer 55 (2012) 1741-1753.

[23] A. Pattamatta, M. Freystein, P. Stephan, A parametric study on phase change heat transfer due to Taylor-Bubble coalescence in a square minichannel, Int. J. of Heat and Mass Transfer 76 (2014) 16-32.

[24] M. Magnini, B. Pulvirenti, J. R. Thome, Numerical investigation of hydrodynamics and heat transfer of elongated bubbles during flow boiling in a microchannel, Int. J. of Heat and Mass Transfer 59 (2013) 451-471.

[25] M. Magnini, B. Pulvirenti, J. R. Thome, Numerical investigation of the influence of leading and sequential bubbles on slug flow boiling within a microchannel, Int. J. of Thermal Sciences 71 (2013) 36-52.

[26] Z. Pan, J. A. Weibel, S. V. Garimella, A saturated-interface-volume phase change model for simulating flow boiling, Int. J. of Heat and Mass Transfer 93 (2016) 945-956.

[27] C. W. Hirt, B. D. Nichols, Volume of fluid (VOF) method for the dynamics of free boundaries, J. of Computational Physics 39 (1981) 201-225.

[28] S. Hardt, F. Wondra, Evaporation model for interfacial flows based on a continuum-field representation of the source terms, J. of Computational Physics 227 (2008) 5871-5895. 
[29] G. M. Lazarek, S. H. Black, Evaporative heat transfer, pressure drop and critical heat flux in a small vertical tube with R113, Int. J. of Heat and Mass Transfer 25 (1982) 945-960.

[30] T. N. Tran, M. W. Wambsganss, D. M. France, Small circular- and rectangular-channel boiling with two refrigerants, Int. J. of Multiphase Flow 22 (1996) 485-498.

[31] Y.-Y. Yan, T.-F. Lin, Evaporation heat transfer and pressure drop of refrigerant R134a in a small pipe, Int. J. of Heat and Mass Transfer 41 (1998) 4183-4194.

[32] Z. Y. Bao, D. F. Fletcher, B. S. Haynes, Flow boiling heat transfer of Freon R11 and HCFC123 in narrow passages, Int. J. of Heat and Mass Transfer 43 (2000) 3347-3358.

[33] D. Shiferaw, X. Huo, T. G. Karayiannis, D. B. R. Kenning, Examination of heat transfer correlations and a model for flow boiling of R134a in small diameter tubes, Int. J. of Heat and Mass Transfer 50 (2007) 51775193.

[34] S. S. Bertsch, E. A. Groll, S. V. Garimella, Effects of heat flux, mass flux, vapor quality, and saturation temperature on flow boiling heat transfer in microchannels, Int. J. of Multiphase Flow 35 (2009) 142-154.

[35] B. Sumith, F. Kaminaga, K. Matsumura, Saturated flow boiling of water in a vertical small diameter tube, Experimental Thermal and Fluid Science 27 (2003) 789-801. 
[36] T.-H. Yen, M. Shoji, F. Takemura, Y. Suzuki, N. Kasagi, Visualization of convecting boiling heat transfer in single microchannels with different shaped cross-sections, Int. J. of Heat and Mass Transfer 49 (2006) 38843894.

[37] B. Agostini, J. R. Thome, M. Fabbri, B. Michel, D. Calmi, U. Kloter, High heat flux flow boiling in silicon multi-microchannels - Part I: Heat transfer characteristics of refrigerant R236fa., Int. J. of Heat and Mass Transfer 51 (2008) 5400-5414.

[38] B. Agostini, J. R. Thome, M. Fabbri, B. Michel, D. Calmi, U. Kloter, High heat flux flow boiling in silicon multi-microchannels - Part II: Heat transfer characteristics of refrigerant R245fa., Int. J. of Heat and Mass Transfer 51 (2008) 5415-5425.

[39] C. L. Ong, J. R. Thome, Flow boiling heat transfer of R134a, R236fa and R245fa in a horizontal $1.030 \mathrm{~mm}$ circular channel, Experimental Thermal and Fluid Science 33 (2009) 651-663.

[40] L. Consolini, J. R. Thome, A heat transfer model for evaporation of coalescing bubbles in microchannel flow, Int. J. of Heat and Fluid Flow 31 (2010) 115-125.

[41] E. Costa-Patry, J. Olivier, B. Michel, J. R. Thome, Two-phase flow of refrigerants in $85 \mu \mathrm{m}$-wide multimicrochannels: Part II - Heat transfer with 35 local heaters, Int. J. of Heat and Fluid Flow 32 (2011) 464-476.

[42] C. L. Ong, J. R. Thome, Macro-to-microchannel transition in two- 
phase flow: Part 2 - flow boiling heat transfer and critical heat flux, Experimental Thermal and Fluid Science 35 (2011) 873-886.

[43] R. Revellin, V. Dupont, T. Ursenbacher, J. R. Thome, I. Zun, Characterization of diabatic two-phase flows in microchannels: Flow parameter results for R-134a in a $0.5 \mathrm{~mm}$ channel, Int. J. of Multiphase Flow 32 (2006) 755-774.

[44] C. L. Ong, J. R. Thome, Macro-to-microchannel transition in two-phase flow: Part 1 - two-phase flow patterns and film thickness measurements, Experimental Thermal and Fluid Science 35 (2011) 37-47.

[45] S. R. Rao, Y. Peles, Spatiotemporally resolved heat transfer measurements for flow boiling in microchannels, Int. J. of Heat and Mass Transfer 89 (2015) 482-493.

[46] V. Dupont, J. R. Thome, A. M. Jacobi, Heat transfer model for evaporation in microchannels. Part II: comparison with the database, Int. J. of Heat and Mass Transfer 47 (2004) 3387-3401.

[47] P. A. Walsh, E. J. Walsh, Y. S. Muzychka, Heat transfer model for gas-liquid slug flows under constant heat flux, Int. J. of Heat and Mass Transfer 53 (2010) 3193-3201.

[48] X. Huo, L. Chen, Y. S. Tian, T. G. Karayiannis, Flow boiling and flow regimes in small diameter tubes, Applied Thermal Engineering 24 (2004) 1225-1239.

[49] K.-I. Choi, A. S. Pamitran, C.-Y. Oh, J.-T. Oh, Boiling heat transfer 
of R22, R134a, and CO2 in horizontal smooth microchannels, Int. J. of Refrigeration 430 (2007) 1336-1346.

[50] D. Shiferaw, T. G. Karayiannis, D. B. R. Kenning, Flow boiling in a $1.1 \mathrm{~mm}$ tube with R134a: Experimental results and comparison with model, Int. J. of Thermal Sciences 48 (2009) 331-341.

[51] Y. Liu, D. F. Fletcher, B. S. Haynes, On the importance of upstream compressibility in microchannel boiling heat transfer, Int. J. of Thermal Sciences 58 (2013) 503-512.

[52] A. M. Jacobi, J. R. Thome, Heat transfer model for evaporation of elongated bubble flows in microchannels, ASME J. of Heat Transfer 124 (2002) 1131-1136.

[53] S. Saitoh, H. Daiguji, E. Hihara, Effect of tube diameter on boiling heat transfer of R134a in horizontal small-diameter tubes, Int. J. of Heat and Mass Transfer 48 (2005) 4973-4984.

[54] J. U. Brackbill, D. B. Kothe, C. Zemach, A continuum method for modeling surface tension, J. of Computational Physics 100 (1992) 335354.

[55] I. Tanasawa, Advances in condensation heat transfer, in: J. P. Hartnett, T. F. Irvine (Eds.), Advances in Heat Transfer, Academic Press, San Diego, 1991.

[56] M. Magnini, CFD modeling of two-phase boiling flows in the slug flow regime with an interface capturing technique, Ph.D. thesis, Alma Mater Studiorum - Università di Bologna, Bologna, Italy, 2012. 
[57] D. L. Youngs, Time-dependent multi-material flow with large fluid distortion, in: K. W. Morton, M. J. Baines (Eds.), Numerical Methods for Fluid Dynamics, Academic Press, 1982, pp. 273-285.

[58] R. I. Issa, Solution of the implicitly discretized fluid flow equations by operator-splitting, J. of Computational Physics 62 (1985) 40-65.

[59] Y. Han, N. Shikazono, Measurement of the liquid film thickness in microtube slug flow, Int. J. of Heat and Mass Transfer 30 (2009) 842853.

[60] A. Scammell, J. Kim, Heat transfer and flow characteristics of rising taylor bubbles, Int. J. of Heat and Mass Transfer 89 (2015) 379-389.

[61] M. Magnini, J. R. Thome, Computational study of saturated flow boiling within a microchannel in the slug flow regime, ASME J. of Heat Transfer 138 (2016) 021502.

[62] Y. Han, N. Shikazono, The effect of bubble acceleration on the liquid film thickness in micro tubes, Int. J. of Heat and Fluid Flow 31 (2010) 630-639.

[63] N. Borhani, B. Agostini, J. R. Thome, A novel time strip flow visualization technique for investigation of intermittent dewetting and dryout in elongated bubble flow in a microchannel evaporator, Int. J. of Heat and Mass Transfer 53 (2010) 4809-4818.

[64] F. P. Bretherton, The motion of large bubbles in tubes, J. of Fluid Mechanics 10 (1961) 166-188. 
[65] S. Khodaparast, M. Magnini, N. Borhani, J. R. Thome, Dynamics of isolated confined air bubbles in liquid flows through circular microchannels: an experimental and numerical study, Microfluid Nanofluid 19 (2015) 209-234.

[66] J. S. Chen, Correlations for boiling heat transfer to saturated fluids in convective flow, Industrial \& Engineering Chemistry Process Design and Development 5 (1966) 322-329.

[67] G. I. Taylor, Deposition of a viscous fluid on the wall of a tube, J. of Fluid Mechanics 10 (1960) 161-165.

[68] P. Aussillous, D. Quéré, Quick deposition of a fluid on the wall of a tube, Physics of Fluids 12 (2000) 2367-2371.

[69] F. Fairbrother, A. E. Stubbs, The bubble-tube method of measurement, J. of Chemical Society 1 (1935) 527-529.

[70] Q. He, Y. Hasegawa, N. Kasagi, Heat transfer modelling of gas-liquid slug flow without phase change in a micro tube, Int. J. of Heat and Fluid Flow 31 (2010) 126-136. 